\title{
réponse sismique d'une couche de vase molle
}

\author{
par \\ A. Pecker \\ J.-P. Walter \\ Géodynamique et structure \\ J. Sigismond \\ E. D. F.-Septen
}

Dans le cadre d'une étude générale de faisabilité relative aux fondations sur pieux d'ouvrages nucléaires, il est apparu important d'étudier les caractéristiques de la réponse au séisme, en l'absence d'ouvrages, du site envisagé. Les stratigraphies envisagées sont constituées d'une couche de sol de faibles caractéristiques mécaniques (vase) surmontant une couche résistante sur laquelle viendraient s'appuyer les pieux.

En l'absence d'un nombre suffisant d'enregistrements réels de séismes en surface de telles couches de sol, il a été décidé d'effectuer l'étude sous un aspect numérique. Les résultats de calcul obtenus ont ensuite été comparés aux quelques enregistrements réels disponibles.

A l'occasion de cette étude deux modèles de comportement de sol ont été étudiés: le modèle viscoélastique linéaire équivalent qui a fait l'objet d'utilisations intensives dans le domaine du génie parasismique et un modèle de sol non linéaire (modèle de Ramberg-Osgood).

\section{Position du problème}

Un séisme donne naissance au foyer, situé à quelques kilomètres sous la surface de la terre, à un train d'ondes. Ces ondes vont se propager jusqu'au voisinage de la surface terrestre. Le problème à résoudre (fig. 1) est celui de la détermination dans les couches de sol proches de la surface du champ des déplacements u, des vitesses ú, des accélérations ü et des contraintes développées lors du passage des ondes sismiques. La solution de ce problème comporte les étapes suivantes:

- mise en équation du problème,

- choix de la loi de comportement du sol,

- détermination des conditions aux limites,

- mise en cuvre d'un schéma d'intégration numérique.

\subsection{Mise en équation du problème}

Les ondes émises au foyer se propagent vers la surface en traversant des terrains de moins en moins résistants. Elles subissent de ce fait des réfractions successives qui modifient leur direction de propagation initiale en la rendant subverticale. Dans le cas extrême de la figure 1 où une couche de faibles caractéristiques surmonte directement une couche très résistante, la direction de propagation des ondes devient quasiment verticale dans la couche supérieure. La réfraction des ondes à l'interface sol-rocher donne naissance à des ondes de cisaillement polarisées $\mathrm{SH}$ et $\mathrm{SV}$ et à des ondes de compression. II est alors justifié d'admettre que le mouvement horizontal résulte de la seule propagation verticale des ondes de cisaillement SH. Dans ces conditions, et pour une stratigraphie horizontale, le seul mouvement induit par le passage de l'onde $\mathrm{SH}$ est un mouvement horizontal d'amplitude $u(t)$. Désignant par :

- $\rho$ la masse volumique du sol;

- $\tau(\mathrm{t})$ la contrainte de cisaillement développée sur un plan horizontal.

L'équation différentielle régissant le mouvement de la couche de sol s'écrit :

$$
\rho \frac{\partial^{2} u}{\partial t^{2}}=\frac{\partial \tau}{\partial z}
$$

\subsection{Loi de comportement du sol}

Un échantillon de sol soumis au laboratoire à des cycles de chargement cyclique présente une courbe effort-déformation analogue à celle de la figure 2. On distingue une courbe de premier chargement (trait tireté) et l'apparition, lors de décharges et recharges successives, d'une boucle d'hystérésis. Plus la déformation maximale atteinte au cours du cycle augmente plus le module de cisaillement sécant (pente de la 


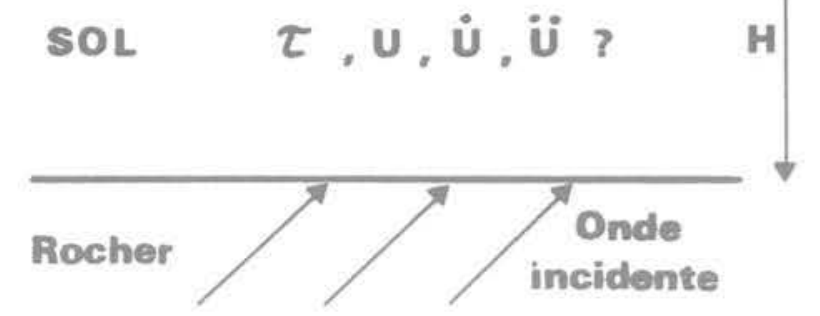

Fig. 1 Définition du problème

droite joignant l'origine à l'extrémité de la boucle) diminue. Cela signifie que sous chargement cyclique, tout comme sous chargement statique, le sol a un comportement non linéaire. L'aire de la boucle d'hystérésis, qui caractérise l'énergie dissipée par le matériau au cours du chargement, augmente lorsque la déformation maximale atteinte au cours du cycle augmente. Enfin, on constate expérimentalement que la forme de la boucle d'hystérésis ne dépend pas de la fréquence de la sollicitation appliquée, ce qui signifie que l'amortissement du sol est de nature purement hystérétique et non d'origine visqueuse.

Pour rendre compte de ce type de comportement, nous avons choisi deux modèles, l'un linéaire, l'autre non linéaire, dont les caractéristiques principales sont rappelées ci-dessous.

\subsubsection{Modèle viscoélastique linéaire équivalent}

Dans le modèle viscoélastique linéaire le matériau est représenté d'un point de vue rhéologique par un ressort de raideur $G$ et par un amortisseur de coefficient $\eta$. En plaçant ces deux éléments en parallèle on obtient le modèle de Kelvin-Voigt dont la loi de comportement s'écrit :

$$
\tau=\mathrm{G} \gamma+\eta \dot{\gamma}
$$

où $\gamma$ et $\dot{\gamma}$ représentent la déformation et la vitesse de déformation. Dans ce type de modélisation, le coefficient $\eta$ est en général pris constant, ce qui donne naissance à un amortissement d'origine visqueuse, en contradiction avec les données expérimentales. Pour tenir compte du comportement hystérétique du sol, il est possible d'introduire, selon une procédure décrite par Jacobsen (1930), un amortissement visqueux dit "équivalent " permettant de ramener l'étude d'un matériau hystérétique à celui d'un matériau viscoélastique. Dans cette procédure, l'équivalence se fait sur l'égalité des énergies dissipées dans le matériau réel et, à résonance, dans le modèle viscoélastique. On définit ainsi un pourcentage d'amortissement critique équivalent $\beta$ :

$$
\beta=\frac{1}{4 \pi} \frac{\Delta W}{W}=\frac{1}{2 \pi} \frac{\Delta W}{G_{s} \gamma^{2} \max }
$$

où $\Delta W$ (aire de la boucle) représente l'énergie totale dissipée pendant un cycle et W l'énergie élastique emmagasinée au cours du même cycle. L'introduction d'un module de cisaillement complexe G permet d'écrire la loi de comportement sous sollicitation harmonique sous la forme:

$$
\tau=G^{*} \gamma=G^{*} \frac{\partial u}{\partial Z}
$$

Le module $G$ est fonction des caractéristiques $G$ et $\beta$ du matériau. Le modèle de ce type le plus couramment utilisé est celui développé par Lysmer (1975) :

$$
\mathrm{G}^{*}=\mathrm{G}_{\mathrm{s}}\left[1-2 \beta^{2}+2 i \beta \sqrt{1-\beta^{2}}\right] .
$$

Ce type de modèle permet de rendre compte des caractéristiques dissipatives du sol. La non-linéarité n'est prise en compte que de façon approchée à l'aide d'un processus itératif (paragraphe 1.4.1). Le principal avantage de ce type de modèle est sa simplicité : la loi de comportement s'écrit formellement de façon équivalente à celle de l'élasticité linéaire. II permet de calculer de façon satisfaisante les accélérations et contraintes maximales induites, tout au moins pour des profils de sol présentant des caractéristiques usuelles (Martin, 1975). Un des buts de l'étude était de tester sa validité pour des profits de sols mous.

\subsubsection{Modèle non linéaire}

Une des limitations principales du modèle viscoélastique linéaire équivalent est son incapacité à calculer les déformations permanentes résultant d'une sollicitation cyclique. Parmi le grand nombre de modèles non linéaires développés par divers auteurs, nous avons retenu le modèle de Ramberg-Osgood dont la validité a été vérifiée expérimentalement pour des matériaux tels que vases et argiles (Pecker-Dupas, 1981). Ce modèle découle des deux lois de Masing (1926) qui postulent que :

- les déformations irréversibles du sol sont d'origine purement plastiques;

- les courbes de recharge et décharge successives se déduisent de la courbe de premier chargement par une affinité d'un facteur 2 sur les échelles horizontale (déformation) et verticale (contrainte).

D'un point de vue rhéologique, Iwan (1967) a montré qu'un matériau satisfaisant aux lois de Masing peut être représenté par un ensemble de ressorts et de frotteurs (fig. 3).

Les lois de comportement traduisant de façon mathématique ces deux postulats différent par l'expression donnée à la courbe de premier chargement. La formulation de Ramberg-Osgood exprime la déformation en fonction de la contrainte sour la forme:

$$
\begin{gathered}
\gamma=f(\tau)=\gamma_{c}+\frac{1}{G_{\max }}\left(\tau-\tau_{c}\right)\left[1+H\left(\frac{\left|\tau-\tau_{c}\right|}{n}\right)\right] \\
H(x)=\alpha\left(\frac{x}{\tau_{y}}\right)^{\mathrm{R}-1}
\end{gathered}
$$

avec :

$\gamma_{c}, \tau_{c} \quad$ : valeur de la contrainte et de la déformation lors de la précédente inversion de la direction du chargement.

n : paramètre de valeur 1 sur la courbe de premier chargement et 2 sur les courbes de recharge et décharge successives.

$\mathrm{G}_{\max } \quad$ : module de cisaillement maximal du sol (pente de la tangente à la courbe de $1^{\text {or }}$ chargement au voisinage de l'origine).

$\alpha, R \quad$ : constantes caractéristiques du matériau.

$\tau_{y} \quad$ : contrainte de cisaillement de référence.

La loi de comportement implique la connaissance de quatre paramètres : $G_{\max }, \alpha, R, \tau_{y}$. Seul $G_{\max }$ peut être déterminé à partir d'essais en place. La connaissance des autres paramètres nécessite la réalisation d'essais 

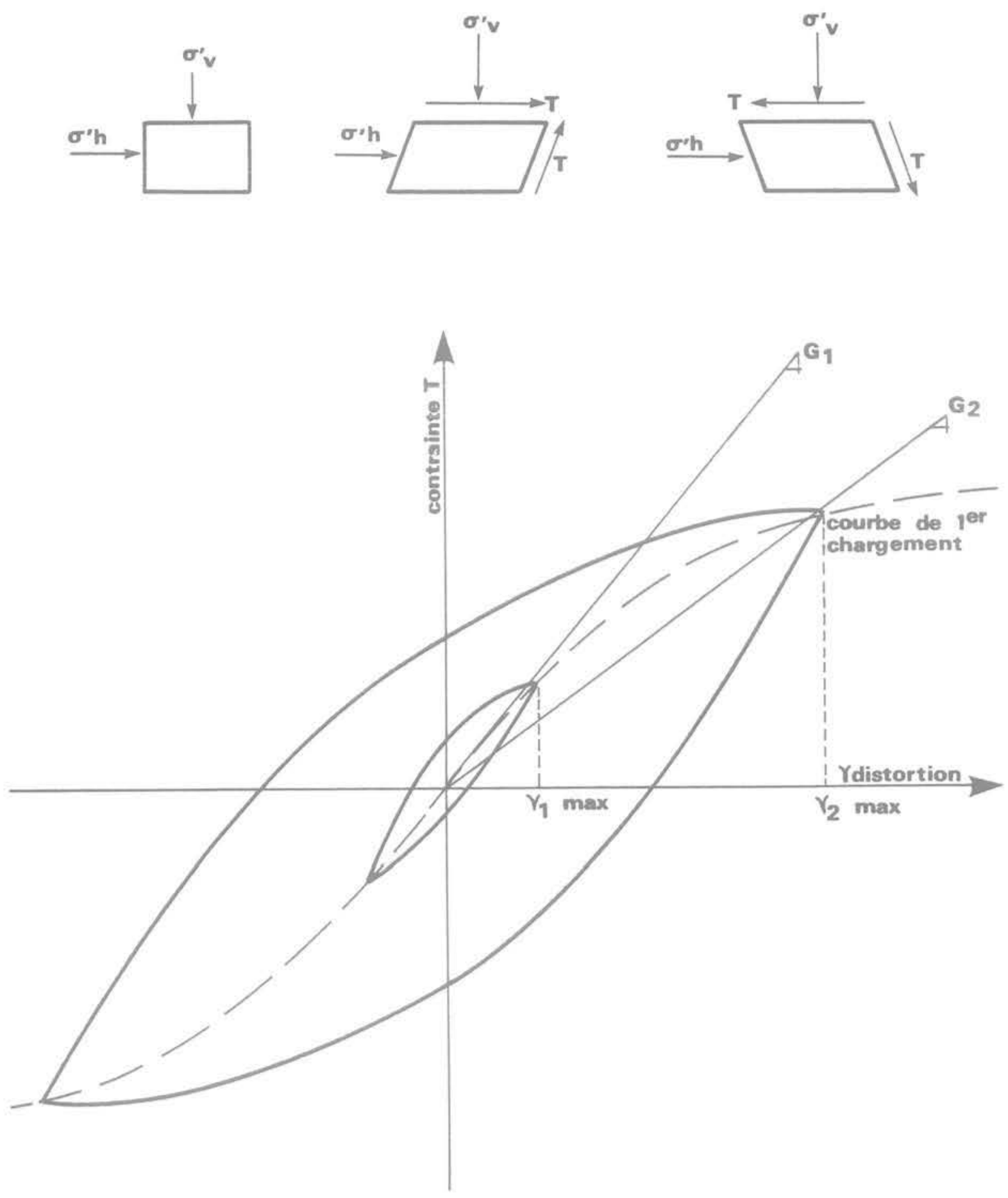

Fig. 2 Courbe effort-déformation cyclique

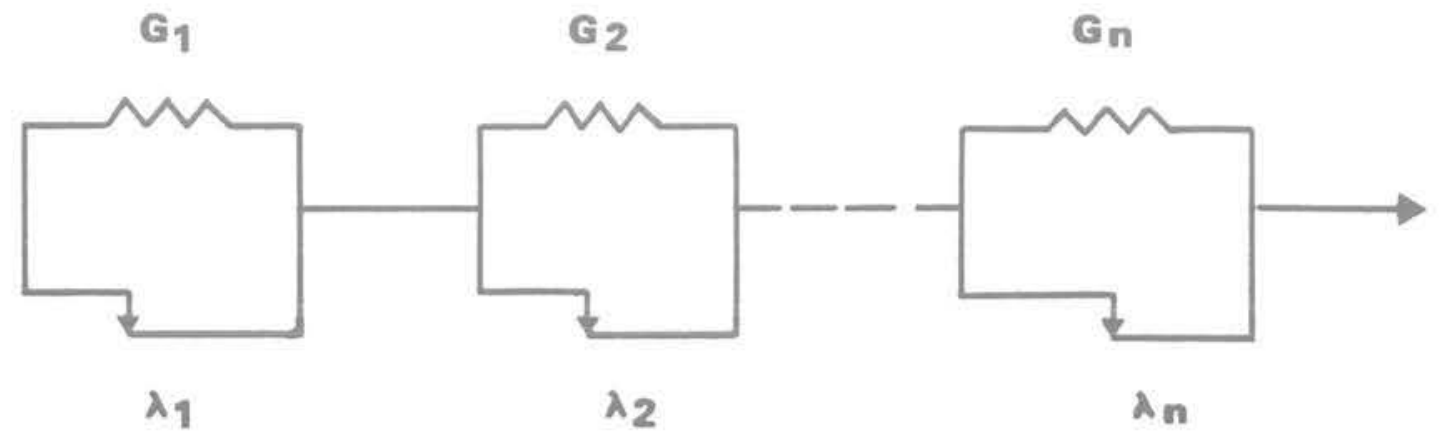

Fig. 3 Modèle plastique.

Modèle de Masing (Iwan, 1967) 

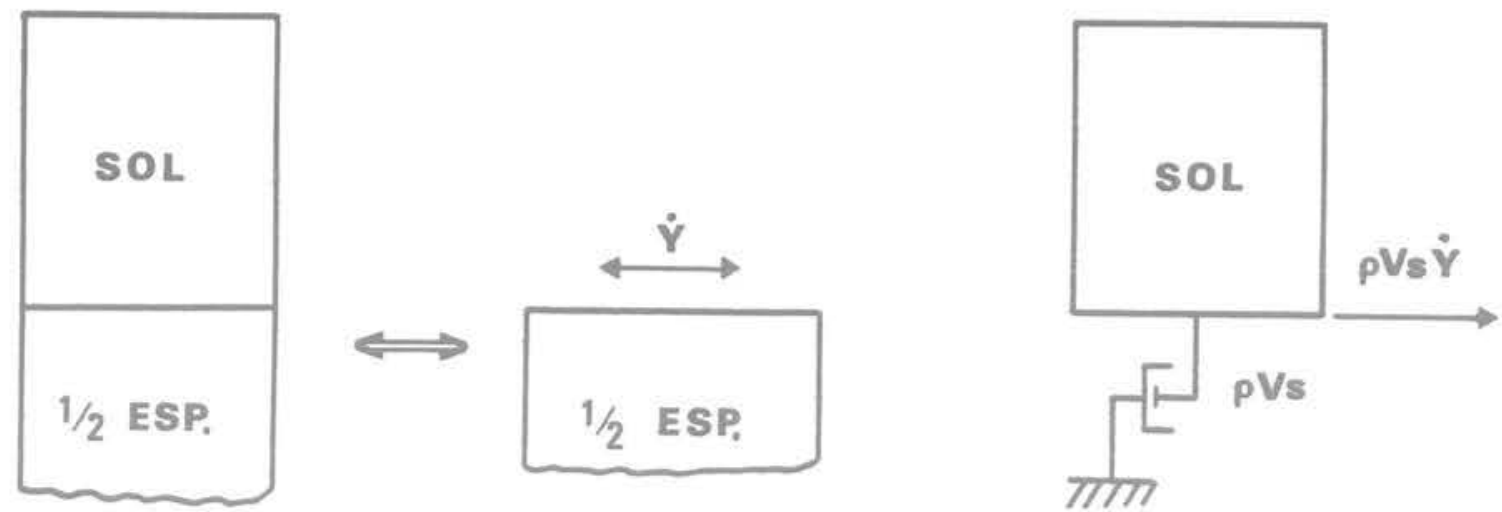

Fig. 4 Condition limite à la base du profil de sol

de laboratoire sous des conditions de contraintes ou déformations contrôlées et pour des sollicitations d'amplitude très faibles à grandes.

II est à noter que les paramètres $G_{s}$ et $\beta$ du modèle viscoélastique linéaire équivalent peuvent être calculés à partir du modèle de Ramberg-Osgood :

$$
\begin{gathered}
G_{s}=\frac{G_{\max }}{1+H(\tau)} \\
\beta=\frac{2}{\pi} \frac{R-1}{R+1}\left[1-\frac{G_{s}}{G_{\max }}\right] .
\end{gathered}
$$

Dans notre étude pour permettre la comparaison entre le modèle linéaire et le modèle non linéaire, nous avons déterminé les paramètres de ce dernier puis calculé ceux du modèle viscoélastique linéaire à l'aide des équations précédentes.

La loi de comportement définie par la relation (4) ou la relation (6) et l'équation de propagation (1) constituent le système d'équations différentielles du mouvement de la couche de sol.

\subsection{Conditions aux limites}

Une condition aux limites évidente du problème est fournie par la condition de surface libre au sommet de la couche de sol:

$$
\left.\tau(\mathrm{t})\right|_{\mathrm{Z}=0}=0 \text {. }
$$

A la base de la couche de sol $(\mathrm{Z}=\mathrm{H})$ le mouvement $u(t, H)$ est supposé connu. En pratique ce mouvement est défini par un accélérogramme jugé représentatif du mouvement d'un rocher lors d'un séisme. Le choix de l'accélérogramme n'est donc pas indifférent. D'autre part, la nature exacte du mouvement du rocher en profondeur n'est pas connue dans la mesure où les mouvements enregistrés lors de séismes réels sont obtenus en surface du sol, c'est-à-dire à une surface libre qui- modifie ce mouvement. Pour pallier à ces difficultés nous avons procédé de la façon suivante :

- le mouvement du rocher est supposé connu non à la base de la colonne de sol mais à un affleurement. En faisant I'hypothèse que le rocher a un comportement élastique linéaire, l'onde incidente du mouvement est la même à la base de la colonne de sol et à l'affleurement. Les mouvements en ces deux points sont cependant différents car ils résultent de la composition de l'onde incidente et d'une onde réfléchie. Cette dernière est identique à l'onde incidente pour l'affleurement (condition de surface libre) mais est modifiée par la propagation à travers la couche de sol suivie de la réflexion à la surface du profil pour le point situé à la base de la colonne de sol. En écrivant cette égalité des amplitudes des ondes incidentes aux deux points considérés, Joyner et Chen (1975) ont montré que, dans l'hypothèse d'ondes de cisaillement à propagation verticale, la condition aux limites exacte à la base de la colonne de sol est donnée par:

$$
\left.\tau(t)\right|_{Z=H}=\rho_{R} V_{R} \dot{y}(t)-\rho_{A} V_{A} \dot{u}(t, Z=H)
$$

où :

$\rho_{A}, V_{A}$ : masse volumique et vitesse de propagation des ondes de cisaillement du rocher.

$y(t) \quad$ : vitesse connue du mouvement de référence à l'affleurement rocheux.

$\dot{u}(t, H)$ : vitesse inconnue à la base de la colonne de sol.

La contrainte donnée par l'équation (11) est la somme d'une contrainte extérieure, connue, appliquée à la base de la colonne de sol, $\rho_{R} \cdot V_{R} \cdot \dot{y}(t)$, et d'une contrainte proportionnelle à la vitesse à la base de cette colonne. Cette dernière est la contrainte que développe un amortisseur de caractéristiques $\rho_{\mathrm{A}} V_{\mathrm{R}}$. On obtient donc une analogie parfaite entre la colonne de sol reposant sur un demi-espace élastique et la même colonne montée sur un amortisseur et soumise à sa base à une contrainte $\rho_{A} V_{A} \dot{y}(t)$ [fig. 4].

Pour choisir un mouvement réaliste $y(t)$ à l'affleurement rocheux nous avons examiné les spectres de réponse proposés par divers auteurs pour des enregistrements sur site rocheux. Ces spectres de réponse à $5 \%$ d'amortissement critique, donnés par Mohraz (1976), Seed-Ugas-Lysmer (1976) et Hayashi et al. (1971), sont rassemblés sur la figure 5 et comparés aux spectres E.D.F. 1973 et U.S.N.R.C. (R. G. 1.60). On constate un très bon accord entre les spectres proposés par les trois groupes d'auteurs. Par ailleurs, le spectre E.D.F, représente une très bonne approximation de ces spectres moyens. L'étude a donc été conduite avec un accélérogramme synthétique dont le spectre de réponse est conforme au spectre E.D.F. Cependant, pour mettre en évidence l'influence du choix du mouvement de référence, certains calculs ont également été effectués avec un accélérogramme dont le spectre de réponse est conforme à celui de la N.R.C. 


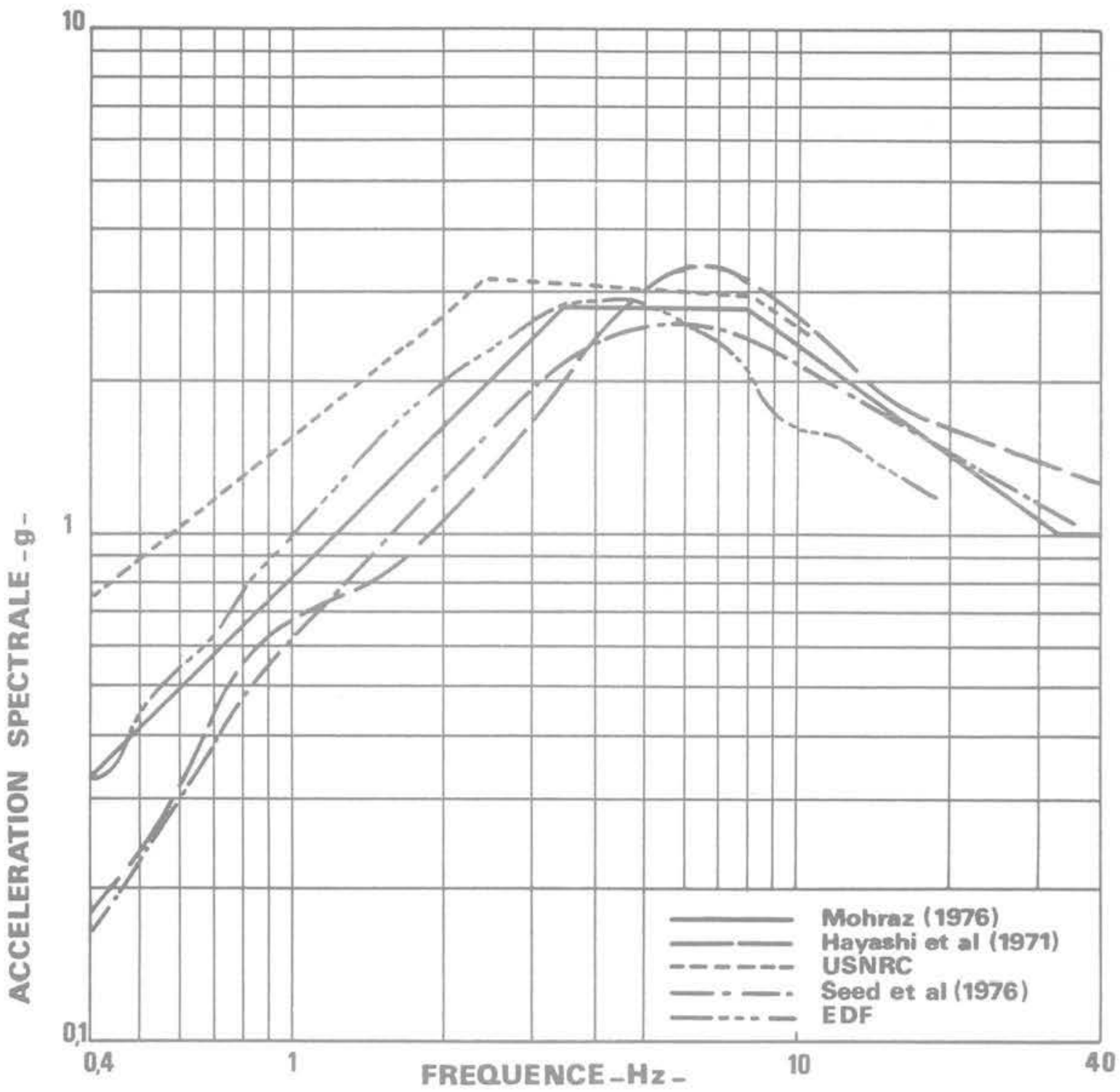

Fig. 5 Spectres de réponse sur rocher.

Pourcentage d'amortissement critique $5 \%$

\subsection{Algorithme d'intégration des équations du mouvement}

L'intégration numérique des équations différentielles du mouvement peut être effectuée soit dans le domaine fréquentiel, soit dans le domaine temps (intégration pas à pas). Ce dernier type d'intégration est obligatoire lorsque la loi de comportement du sol est non linéaire; par contre, le premier avec le développement de la transformation de Fourier rapide (F.F.T.) est particulièrement bien adapté à la loi de comportement viscoélastique linéaire équivalent.

\subsubsection{Intégration dans le domaine fréquentiel}

Le chargement (mouvement de référence) est exprimé sous la forme de la somme d'une série d'harmoniques. La réponse est évaluée pour chaque harmonique et les résultats individuels superposés pour obtenir la réponse globale. Ce type de solution ne peut évidemment être appliqué qu'aux matériaux dont les propriétés sont invariables avec la déformation. Par ailleurs, le chargement pourra être exprimé sous la forme d'une série de Fourier s'il est périodique. Cela ne constitue pas une limitation car pour les problèmes sismiques il suffit d'ajouter à la

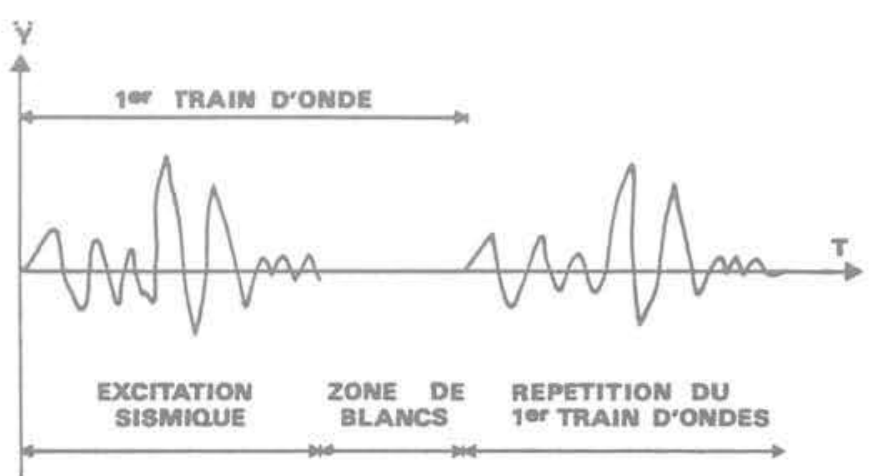

Fig. 6 Intégration dans le domaine fréquence 
perturbation (accélérogramme) une zone de "blancs \# suffisante pour que, compte tenu de l'amortissement interne du matériau, la réponse au $1^{\text {er }}$ train d'excitation soit amortie avant l'arrivée du second train identique au premier (fig. 6).

Le mouvement de référence s'écrivant :

$$
\begin{aligned}
& y(t)=R_{0} \sum_{j=0}^{N / 2} y_{j} e^{i w_{j} t} \\
& w_{j}=\frac{2 \pi j}{T}
\end{aligned}
$$

où :

$\mathrm{i}^{2}:-1$.

$R_{e}$ : partie réelle.

$T$ : durée totale d'un train d'onde (exblancs» compris).

$\mathrm{N} \quad$ : nombre de points de la transformée de Fourier nécessairement de la forme $2^{n}$.

$y_{1}$ : amplitude de l'harmonique j.

La solution est obtenue sous la forme

$$
u(t, Z)=R_{e} \sum_{j=0}^{N / 2} u_{i}(Z) e^{i w_{j} t}
$$

Les inconnues sont les amplitudes $u_{i}$ correspondant aux harmoniques $w_{j}$. Portant l'équation (14) dans l'équation du mouvement (1) et en tenant compte de la loi de comportement du sol [éq. (4) et (5)] on obtient la solution :

$$
u_{j}=E_{j} e^{i k_{j} z}+F_{j} e^{-i k_{j} z}
$$

avec $k_{j}=w_{i} \sqrt{\frac{\rho}{G^{*}}}=$ nombre d'onde.

Le premier terme de l'équation (15) correspond à une onde incidente et le second à l'onde réfléchie. Les constantes d'intégration $E_{j}$ et $F_{j}$, sont obtenues par les conditions aux limites (10) et (11). Dans le cas d'un profil de sol stratifié composé de $n$ couches ayant des propriétés distinctes, il est aisé d'obtenir en écrivant la continuité du déplacement et de la contrainte de cisaillement à l'interface des relations entre les constantes $E_{j}$ et $F_{j}$ de la couche $m$ et celles de la couche $(m+1)$.

Cette méthode de calcul est très efficace du point de vue numérique. Elle a été mise en œuvre par Schnabel et al. (1972) dans le programme de calcul Shake. Utilisée en conjonction avec un processus itératif permettant d'ajuster les caractéristiques $\mathrm{G}_{\mathrm{s}}$ et $\beta$ (éq. 5) du sol avec le niveau de déformation " moyen ", pris usuellement égal aux $2 / 3$ de la déformation maximale, elle permet de rendre compte de façon approchée du caractère non linéaire hystérétique du sol; la solution non linéaire est, dans cette approche, approximée par une succession de solutions linéaires.

\subsubsection{Intégration dans le domaine temps}

Le schéma d'intégration utilisé a été obtenu par Streeter et al. (1973). II est basé sur la résolution de l'équation de propagation par la méthode des caractéristiques. L'équation (1) et la loi de comportement

$$
\tau=f(\gamma)=f\left(\frac{\partial u}{\partial Z}\right)
$$

peuvent être transformées en prenant comme variables la contrainte de cisaillement $\tau$ et la vitesse particulaire $V=u$ u. Le système d'équations différentielles obtenu :

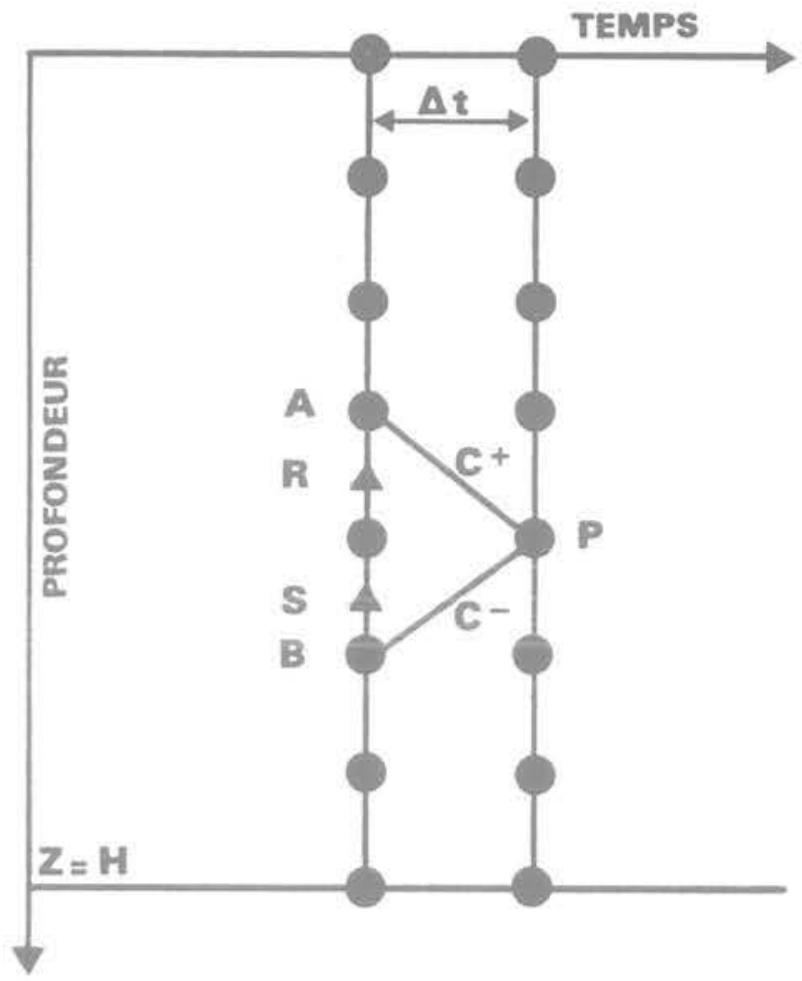

Fig. 7 Intégration le long des lignes caractéristiques

$$
\begin{aligned}
& \frac{\partial \tau}{\partial Z}-\rho \frac{\partial V}{\partial t}=0 \\
& \frac{\partial \tau}{\partial t}-G \frac{\partial V}{\partial Z}=0
\end{aligned}
$$

est un système hyberbolique différentiel du premier ordre dans lequel $\mathrm{G}$ est le module de cisaillement tangent :

$$
\mathrm{G}=\frac{\partial f}{\partial \gamma} \text {. }
$$

Ce système est à résoudre sous les conditions aux limites (10) et (11). D'un point donné dans le plan $(\mathrm{Z}, \mathrm{t})$ sont issues deux lignes caractéristiques $\mathrm{C}^{+}$et $C^{-}$de pente $\pm V_{s}= \pm \sqrt{G / \rho}$ le long desquelles sont vérifiées les relations linéaires suivantes:

$$
\begin{array}{ll}
C^{+} & \frac{d Z}{d t}=V_{s} \\
& \tau-\rho V_{s} V=\text { constante } \\
C^{-} \quad \frac{d Z}{d t}=-V_{s} \\
\\
\tau+\rho V_{s} V=\text { constante }
\end{array}
$$

Le calcul procède alors de la façon suivante (fig. 7) dans le plan $(Z, t)$ : à l'instant $t$ en tous les points du maillage $(A, B, \ldots)$ les valeurs de $\tau$ et $V$ sont connues. Du point $\mathrm{B}$ est issue la caractéristique $\mathrm{C}^{-}$qui au temps $t+d t$ intersecte au point $P$ la caractéristique $\mathrm{C}^{+}$issue de A. Le système d'équations linéaires (20) et (21) permet alors de déterminer $\tau_{p}$ et $V_{p}$. On procède ainsi sur toute la verticale avant de passer au pas de temps suivant. Cette méthode est particulièrement bien adaptée au cas des matériaux linéaires pour lesquels la pente des droites caractéristiques $\left(V_{s}\right)$ est constante. Le maillage vertical étant fixé, le pas de temps en découle. Pour un matériau non linéaire $V_{s}$, et donc la pente des caractéristiques, décroit avec une augmentation de la déformation. Les caractéristiques aboutissant en $\mathrm{P}$ ne sont plus issues de $A$ et $B$ mais de points intermédiaires $R$ et $S$ pour lesquels il faut déterminer les valeurs de $\tau$ et $V$ par interpolation entre celles calculées en A et B. 

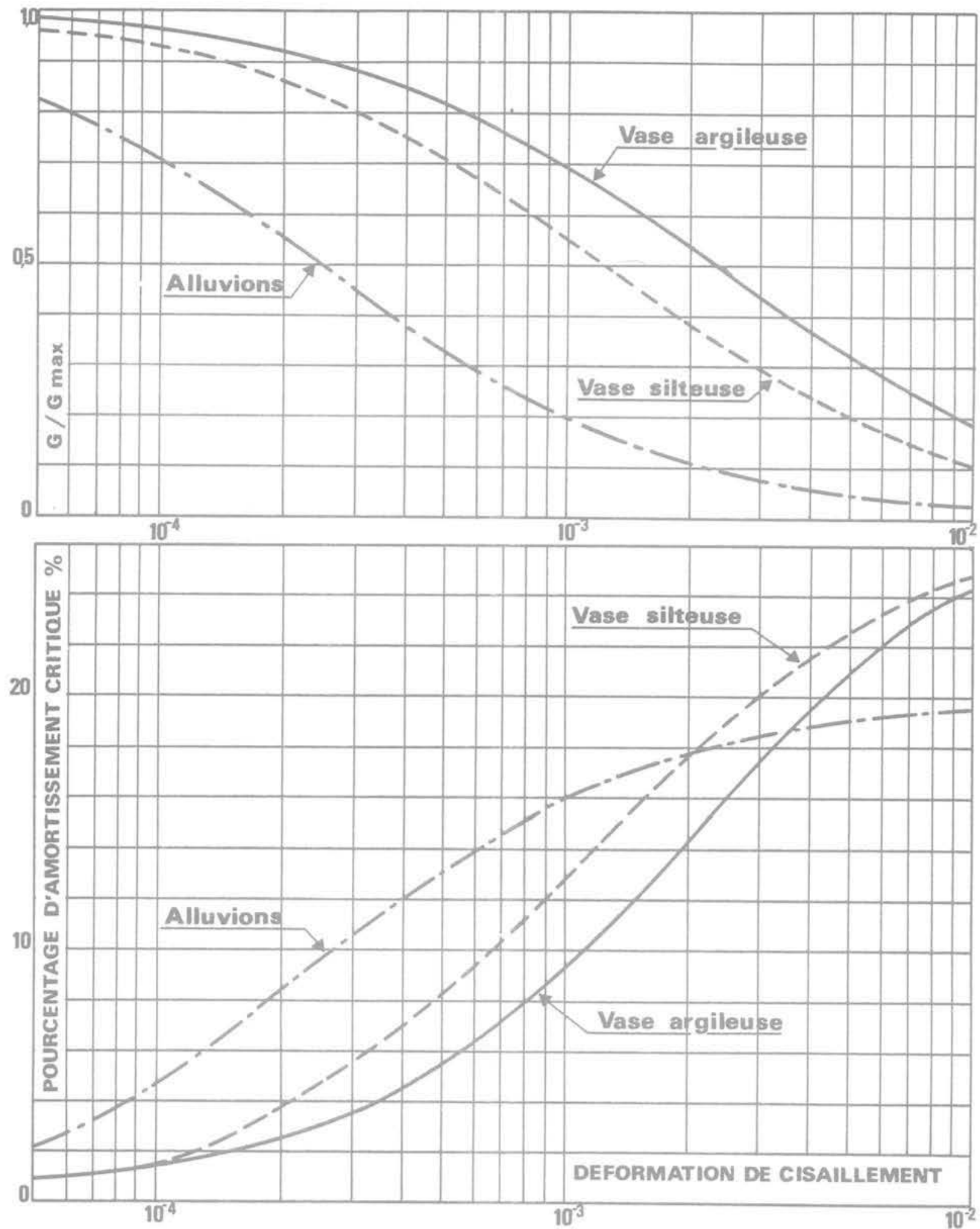

Fig. 8 Variation des caractéristiques dynamiques avec la déformation

\section{Caractéristiques du profil de sol étudié}

Le profil de sol étudié présente des caractéristiques voisines d'un site en bordure de la Loire à proximité de Paimbœuf. Cependant les caractéristiques mécaniques retenues pour l'étude peuvent être légèrement meilleures que les caractéristiques réelles des sols du site. En effet, les valeurs prises en compte constituent des valeurs minimales acceptables pour permettre l'accès et la construction sur ce site et, en tout état de cause, il sera nécessaire préalablement à tous travaux d'améliorer les sols en place pour obtenir ces valeurs.
Depuis la cote 0 NGF, le site tel qu'il a été étudié est constitué sur $13 \mathrm{~m}$ de vase argileuse, puis sur $5 \mathrm{~m}$ de vase silteuse varvée surmontant $2 \mathrm{~m}$ d'alluvions anciennes constituées de sables et graviers. Le rocher sous-jacent est constitué de gneiss, plus ou moins altéré au contact. Les caractéristiques principales des différents terrains sont rassemblées dans le tableau I. Pour les besoins de l'étude, les caractéristiques essentielles sont le poids volumique apparent humide et le module de cisaillement dynamique (ou la vitesse de propagation des ondes de cisaillement). Pour les vases, les valeurs du module ont été déduites d'essais triaxiaux cycliques réalisés en laboratoire sur les 


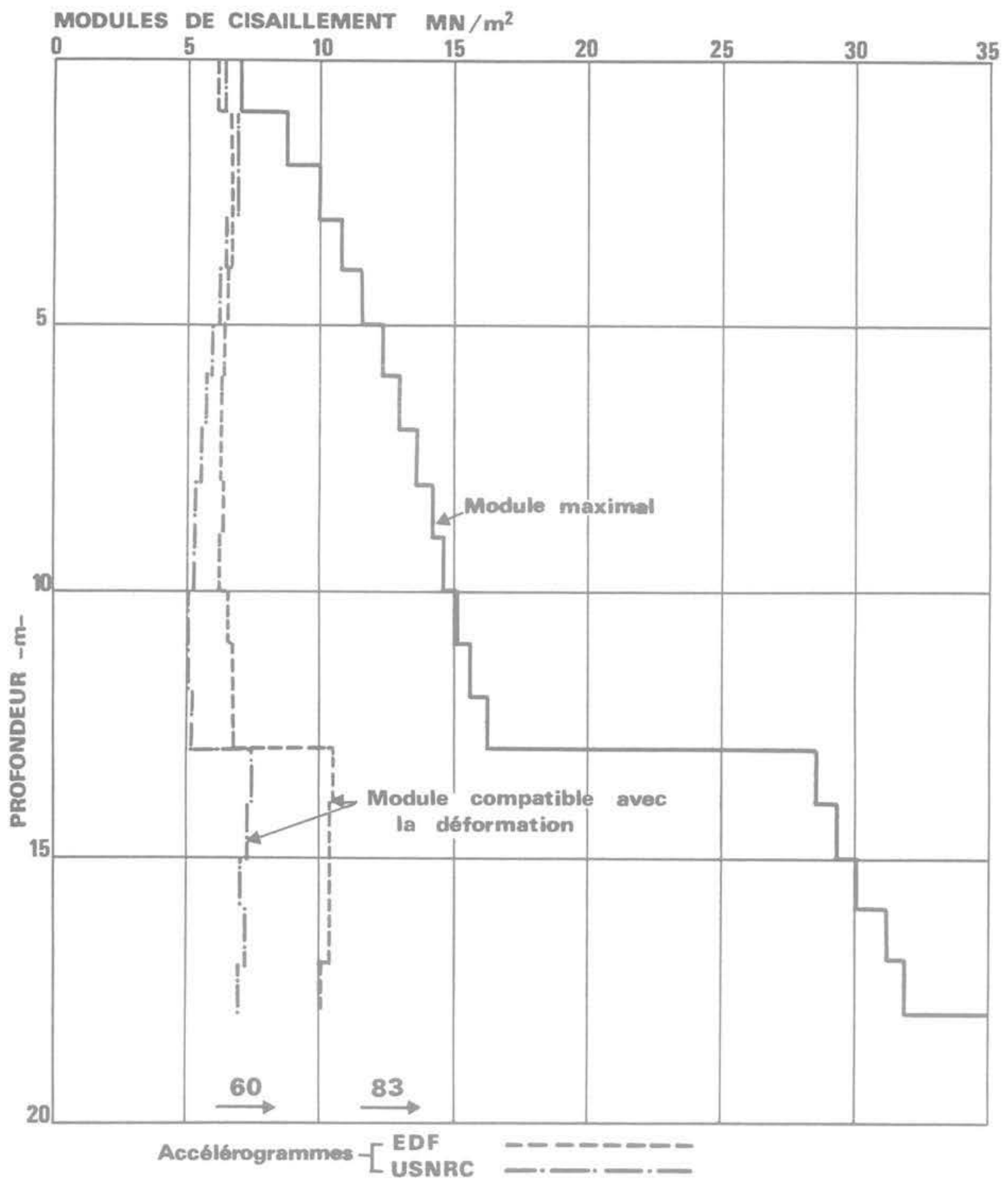

Fig. 9 Variation du module avec la profondeur.

Accélérations à l'affleurement $0,3 \mathrm{~g}$

vases de Montoir de Bretagne situé en face du site sur l'autre rive de la Loire. Pour les alluvions et le rocher, ces valeurs ont été déduites de la littérature. Dans la pratique, compte tenu du contraste très important entre les vitesses de propagation d'ondes dans le rocher $(\sim 1.500 \mathrm{~m} / \mathrm{s})$ et dans les vases (100 à $150 \mathrm{~m} / \mathrm{s}$ ) la valeur exacte de la vitesse dans le rocher n'a aucune influence sur les résultats; ce paramètre n'a donc pas été étudié en détails.

Dans les vases le module de cisaillement maximal est de la forme:

$$
\mathrm{G}_{\max }=K \mathrm{P}_{\mathrm{a}}\left(\frac{\sigma^{\prime} \mathrm{m}}{\mathrm{P}_{\mathrm{a}}}\right)^{\alpha}(\mathrm{OCR})^{\mathrm{k}}
$$

avec :

$\mathrm{K}, \alpha \mathrm{k}$ : paramètres caractéristiques du sol (tableau I)

$\sigma^{\prime} m \quad$ : contrainte moyenne effective

$\mathrm{Pa} \quad$ : pression atmosphérique

OCR : rapport de surconsolidation des sols. Supérieur à 1 puisque initialement le TN est à la cote 7 NGF et que les sols sont supposés améliorés.

La variation du module de cisaillement maximal avec la profondeur est donnée sur la figure 9.

Les courbes de variation du module de cisaillement et du pourcentage d'amortissement du sol avec la déformation (équations 8 et 9) sont représentées sur la figure 8 . 


\section{3 Études paramétriques effectuées}

Ces études ont porté sur les caractéristiques du mouvement sismique de référence, sur la loi de comportement des sols et sur les caractéristiques du profil de sol.

\subsection{Nature du mouvement sismique}

On a essentiellement fait varier le niveau d'accélération à l'affleurement entre $0,20 \mathrm{~g}$ et $0,30 \mathrm{~g}$. Par ailleurs pour mettre en évidence l'influence de la nature du mouvement sismique et insister sur la nécessité de prendre en compte des mouvements réalistes, une étude comparative a été faite à partir de l'accélérogramme synthétique représentant le spectre N.R.C. et à partir de celui représentant le spectre E.D.F. qui est le plus proche d'un accélérogramme de rocher.

Ces études paramétriques sur la nature du mouvement sismique ont été effectuées avec le modèle linéaire équivalent.

\subsection{Loi de comportement du sol}

Pour le niveau d'accélération le plus élevé envisagé $(0,30 \mathrm{~g})$, un calcul non linéaire a été réalisé pour vérifier, dans les conditions les plus sévères, la validité du modèle linéaire équivalent. Compte tenu des comparaisons favorables obtenues, d'autres calculs non linéaires n'ont pas été entrepris.

\subsection{Caractéristiques de la couche de sol}

Pour juger de l'influence des caractéristiques de sol sur la nature du mouvement à la surface, les modules de cisaillement maximaux des trois couches constitutives du profil étudié ont été majorés de $50 \%$ en conservant leurs épaisseurs constantes. Un autre calcul a été effectué en majorant les modules de $50 \%$ et les épaisseurs de couches de $20 \%$ de façon à conserver la fréquence fondamentale, à petites déformations, du profil de sol. Ces calculs ont également été réalisés avec le modèle linéaire équivalent.

\section{Influence de la nature du mouvement sismique}

Pour mettre en évidence l'influence de la nature du mouvement sismique sur les résultats, nous avons choisi de présenter les résultats obtenus pour l'accélération à l'affleurement la plus élevée, soit $0,30 \mathrm{~g}$. Des résultats identiques à ceux-ci ont également été obtenus pour les niveaux d'accélération de $0,20 \mathrm{~g}$ et $0,25 \mathrm{~g}$.

La variation en fonction de la profondeur du module de cisaillement compatible avec la déformation "moyenne" induite est donnée sur la figure 9. La réduction par rapport au module maximal à petites déformations (équation 22) est importante pour les deux accélérogrammes; elle atteint, sous $10 \mathrm{~m}$ de profondeur, un rapport de 1 à 3 , voir 1 à 4 . La réduction est d'environ $30 \%$ plus importante avec l'accélérogramme U.S.N.R.C. qu'avec l'accélérogramme E.D.F.

Le profil d'accélération maximale est donnè en fonction de la profondeur sur la figure 10. Les valeurs obtenues avec les deux accélérogrammes ne sont pas

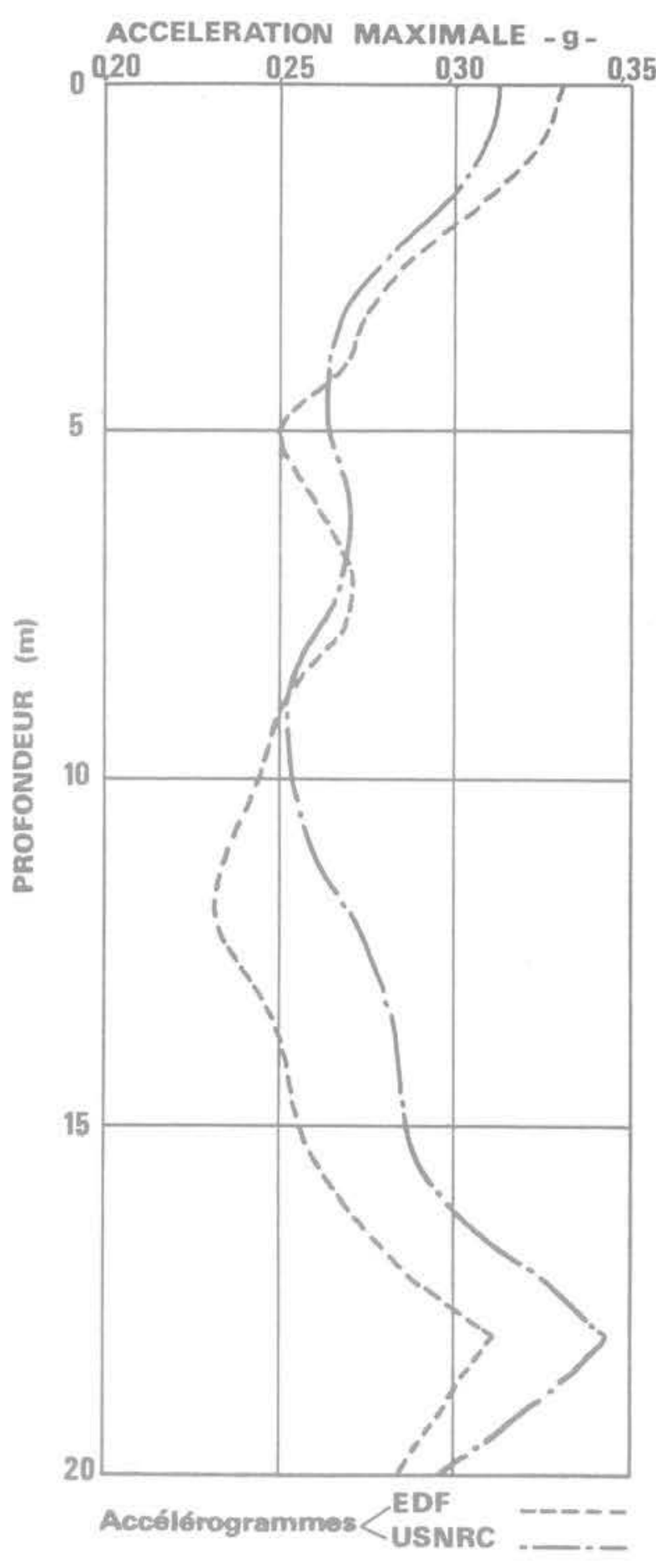

Fig. 10 Variation de l'accélération avec la profondeur. Accélérations à l'affleurement $0,30 \mathrm{~g}$

essentiellement différentes (moins de $10 \%$ d'écart). Cependant, et ce résultat a été obtenu de façon systématique pour les autres calculs, l'accélérogramme U.S.N.R.C. conduit à des accélérations de surface plus faibles que l'accélérogramme E.D.F. La tendance est inversée en profondeur.

La figure 11 donne les spectres de réponse du mouvement à la surface pour un pourcentage d'amortissement critique de $5 \%$. Ces spectres présentent deux pics prononcés au voisinage de $1 \mathrm{~Hz}$ et de $3 \mathrm{~Hz}$ qui correspondent aux deux premières fréquences propres de vibration de la couche de sol. Ces deux pics se retrouvent sur les fonctions de transfert du déplacement (fig. 12). Ils correspondent à des fréquences légèrement plus faibles lorsque 


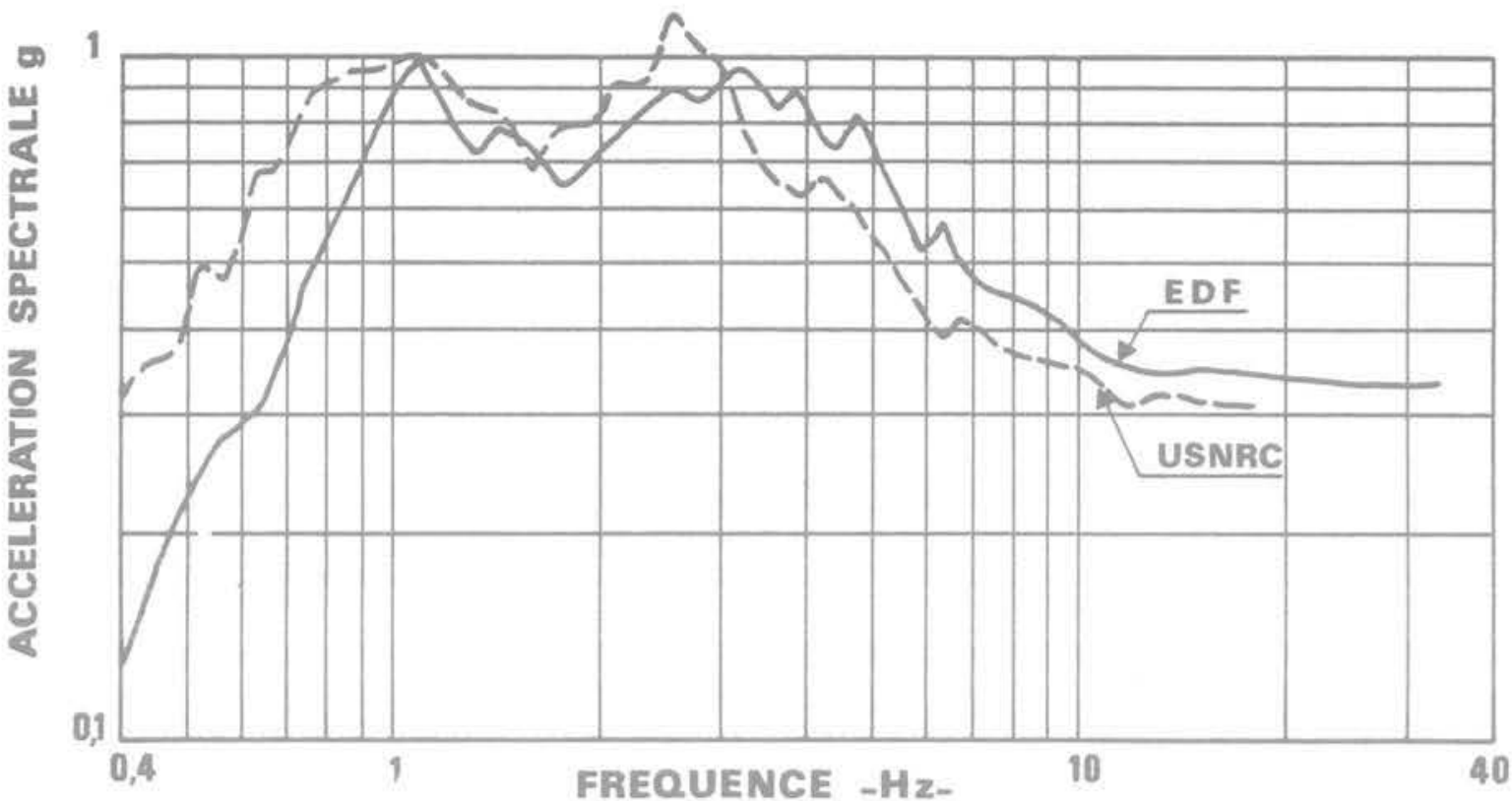

Fig. 11 Spectre de réponse à $5 \%$ d'amortissement critique à la surface du sol. Accélérogramme $0,3 \mathrm{~g}$ à l'affleurement

l'accélérogramme U.S.N.R.C. est utilisé. Ce résultat est conforme à celui de la figure 9 qui a montré que cet accélérogramme conduisait à des modules plus faibles que l'accélérogramme E.D.F.

Pour des fréquences supérieures au hertz, les deux spectres de réponse sont voisins l'un de l'autre. Par contre, aux faibles fréquences, l'accélérogramme U.S.N.R.C. conduit à des accélérations spectrales nettement plus élevées. En rapprochant ces spectres de ceux des accélérogrammes de départ (fig. 5) on constate que, quel que soit l'accélérogramme utilisé, pour les fréquences inférieures à $3 \mathrm{~Hz}$, il y a amplification de l'accélération spectrale; cette amplification est particulièrement marquée au voisinage de la fréquence fondamentale de la couche de sol $(\sim 1 \mathrm{~Hz})$. Au-delà de $3 \mathrm{~Hz}$, il y a atténuation des accélérations spectrales. Cette atténuation est plus marquée avec le spectre U.S.N.R.C. plus riche en hautes fréquences que le spectre E.D.F.

L'ensemble de ces résultats met en évidence les caractéristiques fondamentales de la réponse au séisme d'une couche de vase molle :

- les hautes fréquences du mouvement sont filtrées et les basses fréquences amplifiées;

- les spectres de réponse du mouvement à la surface font apparaître un pic prononcé à la fréquence fondamentale de la couche; un deuxième pic moins marqué apparaît pour la deuxième fréquence propre. Ce résultat montre, et cela est confirmé par l'examen des déplacements, que le mode de vibration fondamental de la couche (quart d'onde) est très nettement prépondérant sur les modes supérieurs.

Par ailleurs, ces résultats mettent en évidence l'influence du choix de la nature du mouvement à l'affleurement. Si ce dernier contient, comme c'est le cas pour l'accélérogramme U.S.N.R.C., des basses fréquences peu représentatives de celles contenues dans un mouvement de rocher, celles-ci seront amplifiées par la couche de sol et conduiront à des mouvements à basse fréquence trop importants. Les déplacements et déformations, gouvernés par les basses fréquences, seront donc surévalués. Ce résultat peut avoir des conséquences importantes pour les ouvrages fondés sur pieux souples suivant la déformation du sol. II explique par ailleurs les résultats des figures 9 et 10 : les modules étant fonction des déformations induites, plus élevées avec l'accélérogramme U.S.N.R.C., sont naturellement plus faibles lorsqu'ils sont évalués avec ce dernier.

De même, le spectre U.S.N.R.C. étant plus riche en hautes fréquences que le spectre E.D.F., il induit des accélérations plus fortes en base de couche; au voisinage de la surface le filtrage plus important des hautes fréquences, lié aux valeurs plus faibles des modules, conduit à des accélérations inférieures à celles calculées avec l'accélérogramme E.D.F. Pour ces raisons et compte tenu des remarques faites au paragraphe 1.3 concernant les caractéristiques de mouvements enregistrés sur rocher, la suite de l'étude a été conduite essentiellement avec l'accélérogramme E. D. F.

\section{Influence du niveau d'accélération à l'affleurement}

Trois niveaux d'accélérations à l'affleurement ont été envisagés : $0,20 \mathrm{~g}, 0,25 \mathrm{~g}$ et $0,30 \mathrm{~g}$. La variation de l'accélération maximale de surface en fonction de celle à l'affleurement est donnée sur la figure 13. Dans la plage des accélérations étudiées, il y a toujours amplification de l'accélération maximale. Cette amplification est plus importante avec l'accélérogramme E.D.F. qu'avec l'accélérogramme U.S.N.R.C. Lorsque le niveau d'accélération à l'affleurement augmente, laccélération maximale de surface croît mais moins rapidement. Le rapport d'amplification entre les deux accélérations décroît et pour la valeur maximale envisagée de $0,30 \mathrm{~g}$, il est voisin de 1 . II est vraisemblable que pour des accélérations plus élevées à l'affleurement, ce rapport deviendra inférieur à 1; il y aura atténuation de l'accélération maximale. Cette particularité est due à la non linéarité du sol et à sa capacité limitée à transmettre des contraintes élevées. A une profondeur donnée la contrainte de cisaillement maximale qu'il peut transmettre est égale à sa résistance au cisaillement sous chargement cyclique. Ce plafonnement de la contrainte se traduit dans le calcul par la diminution du module et l'augmentation de l'amortissement lorsque les sollicitations, donc les déformations induites, 


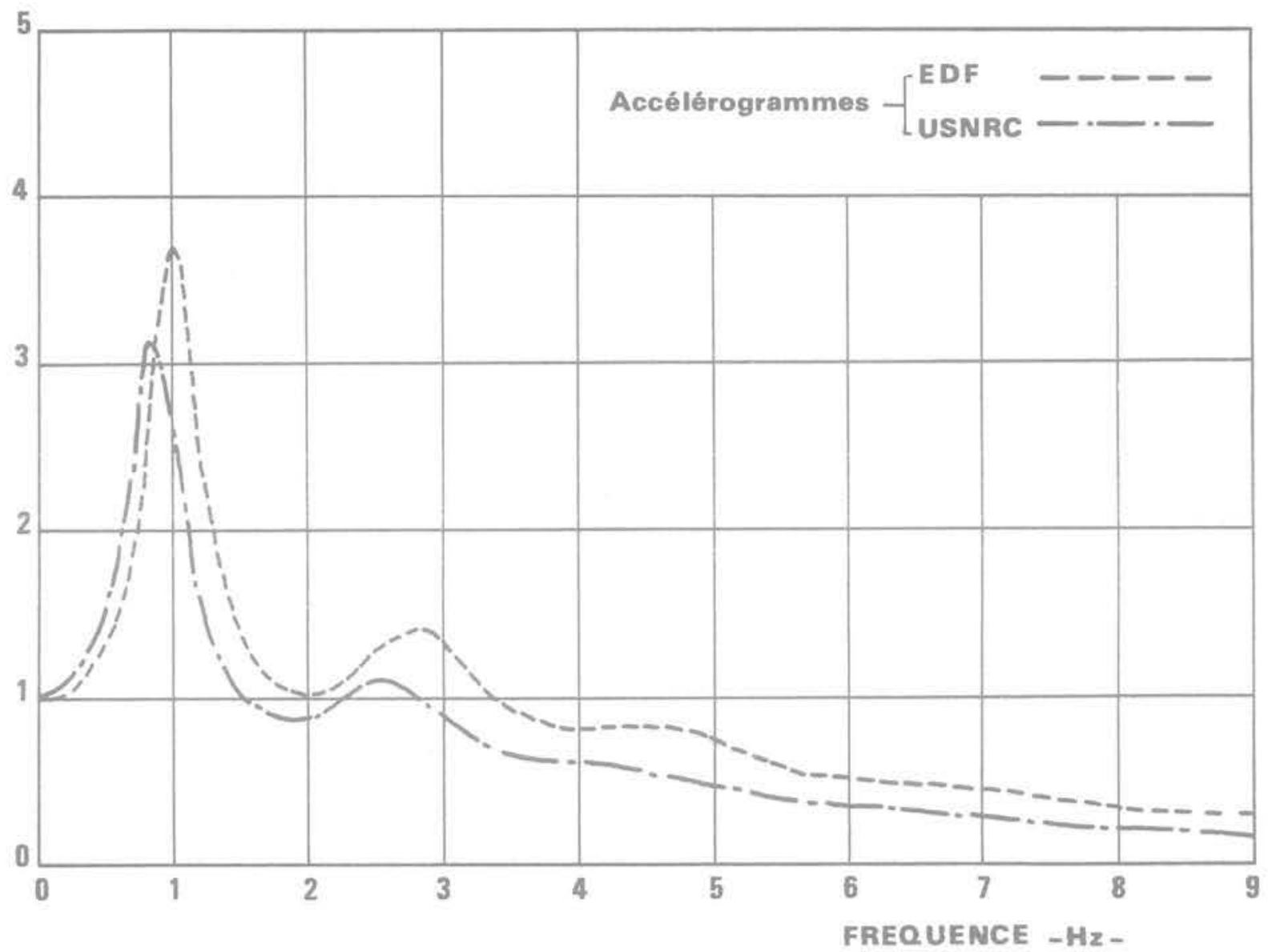

Fig. 12 Fonction de transfert du déplacement entre la base et la surface de la colonne de sol. Accélérations à l'affleurement $0,3 \mathrm{~g}$

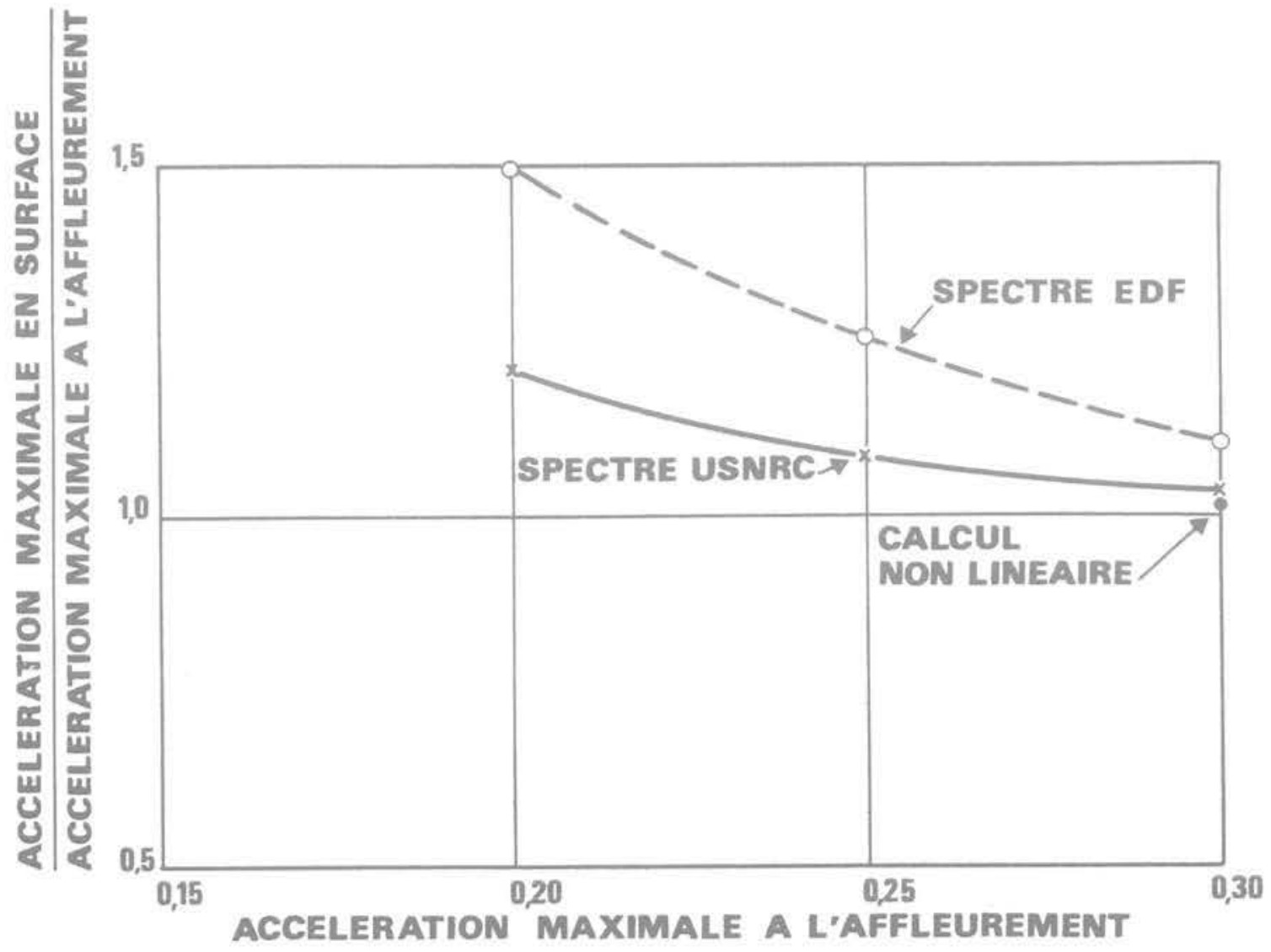

Fig. 13 Variation de l'accélération de surface en fonction de l'accélération à l'affleurement 


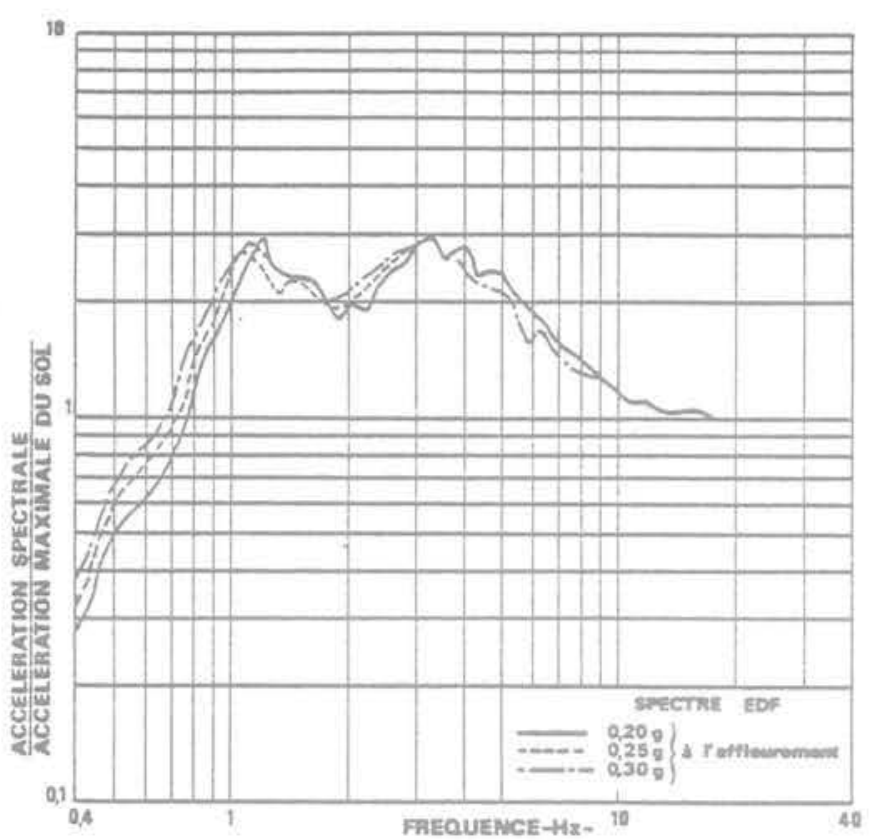

Fig. 14 Spectre de réponse à $5 \%$ d'amortissement critique. Norme à $1 \mathrm{G}$. Surface du sol

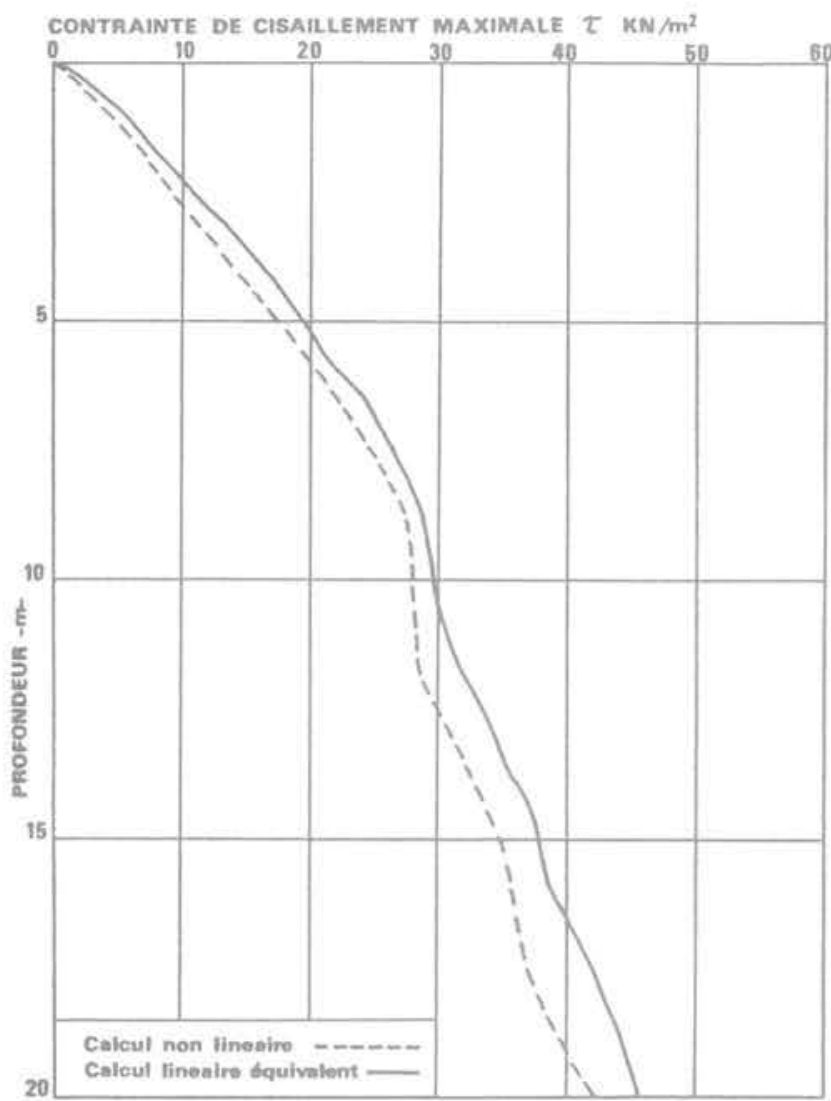

Fig. 15 Variation de la contrainte de cisaillement avec la profondeur.

Spectre EDF 0,3 $\mathrm{g}$ à l'affleurement

augmentent. L'accélération maximale de surface doit donc nécessairement tendre vers une limite fonction des caractéristiques de la couche de sol. Ceci a été observé et obtenu par le calcul pour des niveaux d'accélération plus élevés (Mohammadioun-Pecker, 1983).

Les spectres de réponse du mouvement à la surface sont donnés sur la figure 14 pour $5 \%$ d'amortissement critique. Pour permettre la comparaison les 3 spectres ont été normés par l'accélération maximale de surface. Ils présentent tous les trois les

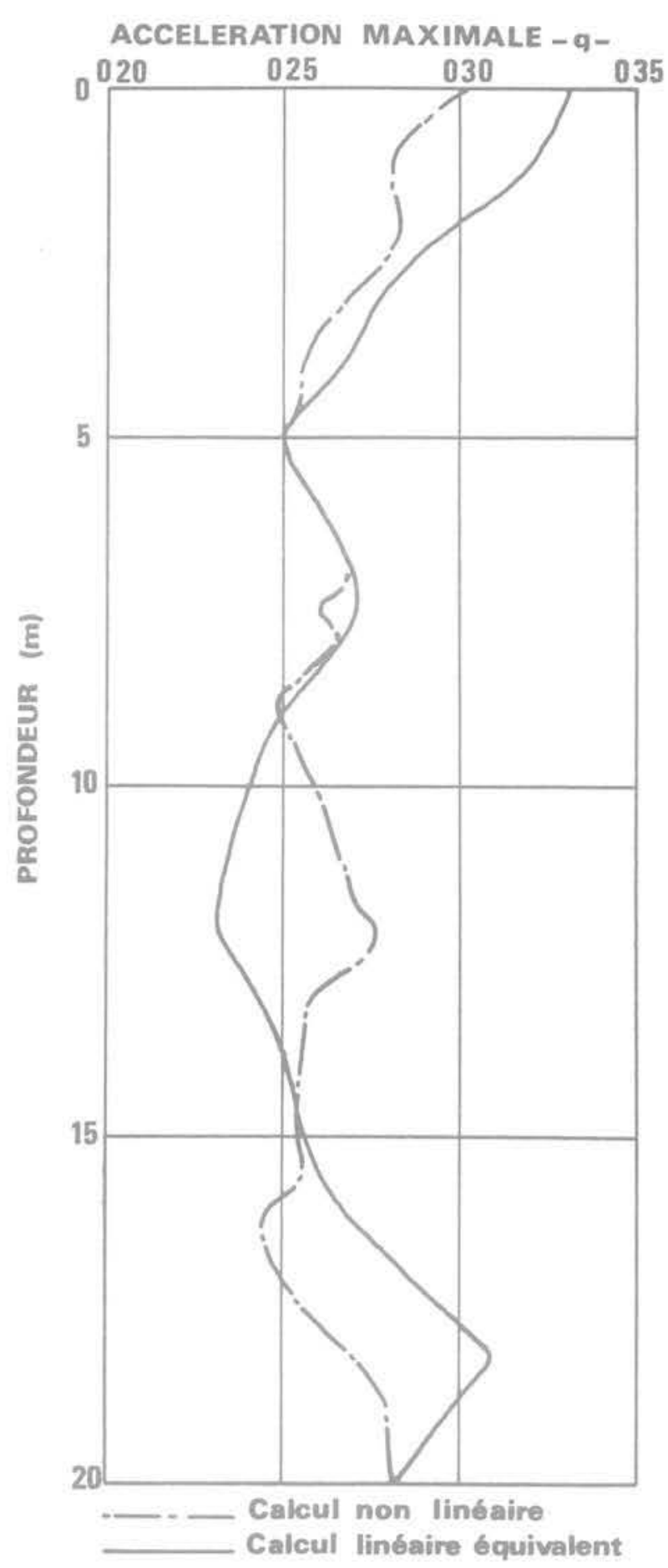

Fig. 16 Variation de l'accélération avec la profondeur. Spectre EDF $0,30 \mathrm{~g}$ à l'affleurement

particularités décrites précédemment à savoir deux pics prononcés avec deux premières fréquences propres de vibration de la couche de sol. Lorsque le niveau d'accélération à l'affleurement croît, les pics se décalent légèrement vers les basses fréquences du fait de la diminution de raideur de la couche due aux sollicitations plus élevées. Pour les fréquences supérieures à la fréquence fondamentale de la couche $(\sim 1 \mathrm{~Hz})$ les formes de spectres sont semblables. Par contre, pour les fréquences inférieures, plus l'accélération est élevée à l'affleurement plus le spectre de réponse à la surface est riche en basses fréquences. Ce résultat met en évidence les limites d'utilisation de spectres de forme normalisée calés au niveau d'accélération approprié. Cette pratique est cependant souvent utilisée (Seed et al. 1976, Mohraz 


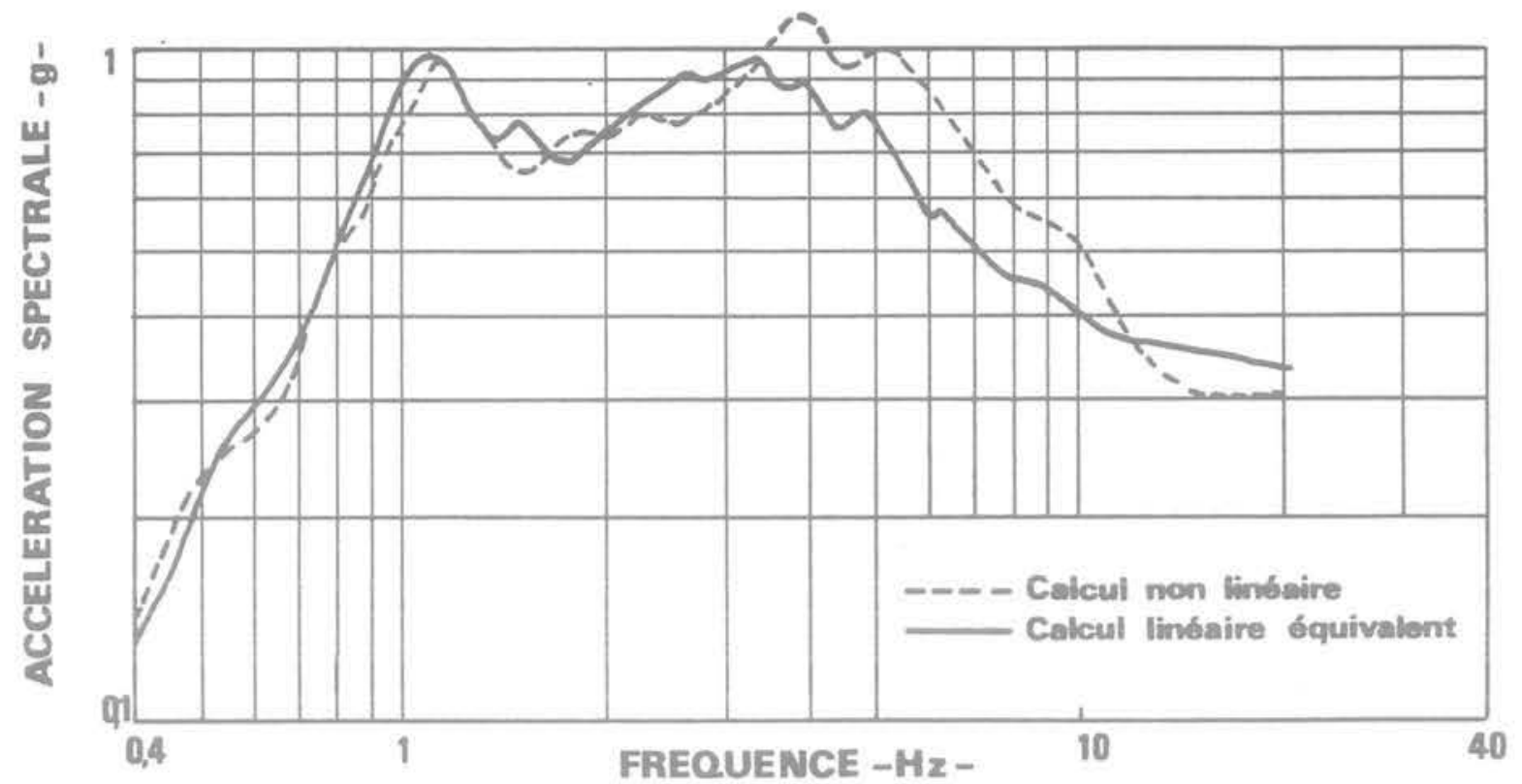

Fig. 17 Spectre de réponse à $5 \%$ d'amortissement critique à la surface du sol. Accélérogramme EDF $0,30 \mathrm{~g}$ à l'affleurement

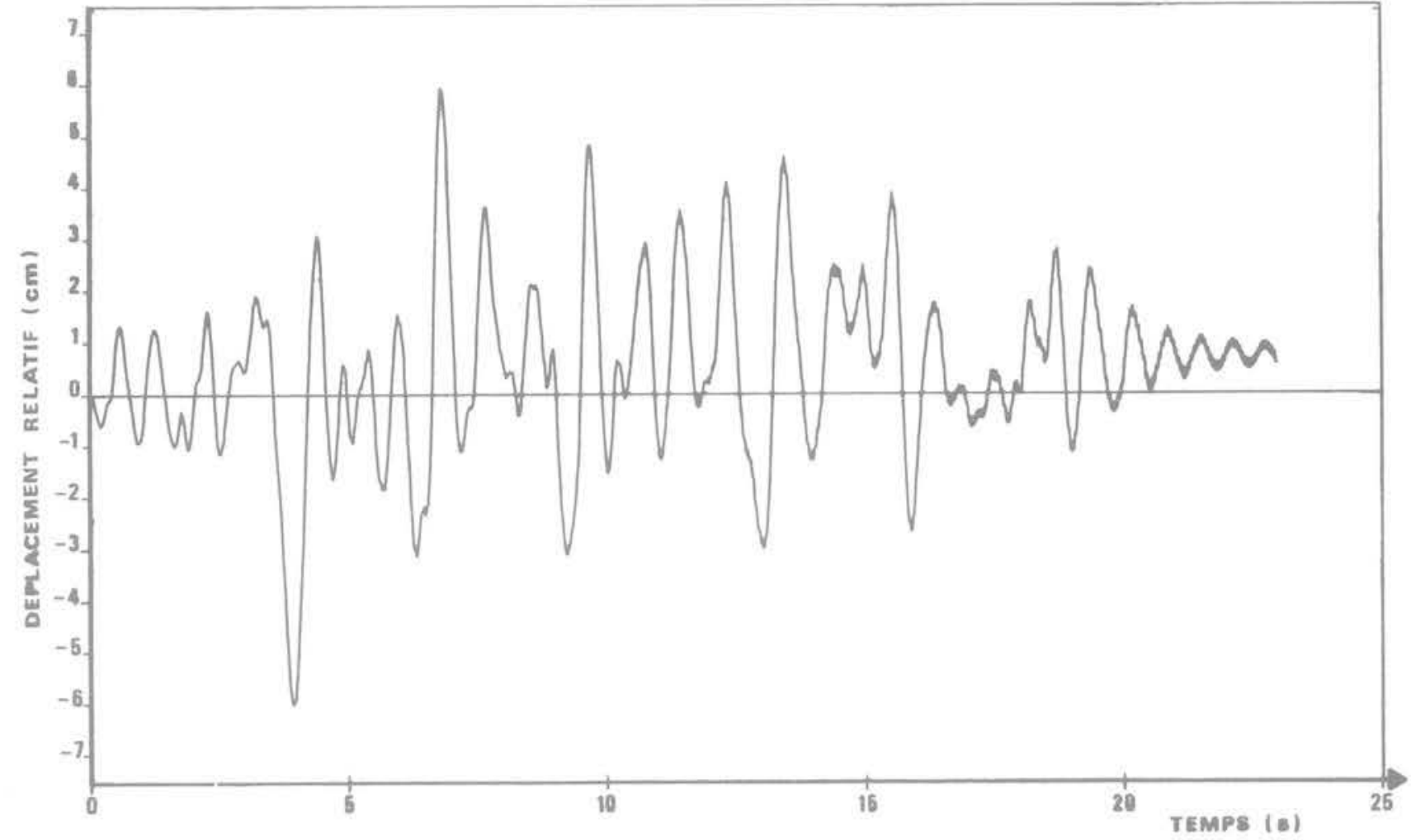

Fig. 18 Déplacement relatif entre la surface et la base du profil de sol

1976...) et est en particulier employée dans la réglementation de la N.R.C. américaine. La pratique française en matière d'ouvrages nucléaires conduit au contraire à des formes de spectre adaptées au niveau sismique (Devillers-Mohammadioun, 1981).

\section{Comparaison entre calcul linéaire équiva- lent et calcul non linéaire}

Les çalculs de réponse dynamique d'un profil de sol sont généralement mis en œuvre avec le modèle linéaire équivalent dans le but d'obtenir les accélérations et contraintes maximales dans le sol. La validité de tels calculs a été testée dans le cas de la sollicitation la plus élevée $(0,30 \mathrm{~g})$ et avec l'accélérogramme E.D.F. Les contraintes de cisaille- ment maximales sont comparées sur la figure 15. L'accord entre les valeurs obtenues par les deux calculs est très bon sur toute la hauteur de la couche; l'écart est inférieur à $10 \%$. Les accélérations maximales sont comparées sur la figure 16; bien que de légers écarts ( 15 à $20 \%$ ) subsistent à certaines profondeurs l'accord peut être jugé satisfaisant d'autant que dans le calcul non linéaire l'accélération est obtenue à partir de la vitesse par dérivation, ce qui constitue une opération numérique très délicate. Les spectres de réponse en surface sont comparés sur la figure 17. Jusqu'à une fréquence de $3 \mathrm{~Hz}$ ils sont parfaitement semblables. Pour les fréquences plus élevées, le calcul non linéaire conduit à des accélérations spectrales plus élevées. En d'autres termes, le calcul linéaire équivalent filtre de façon 


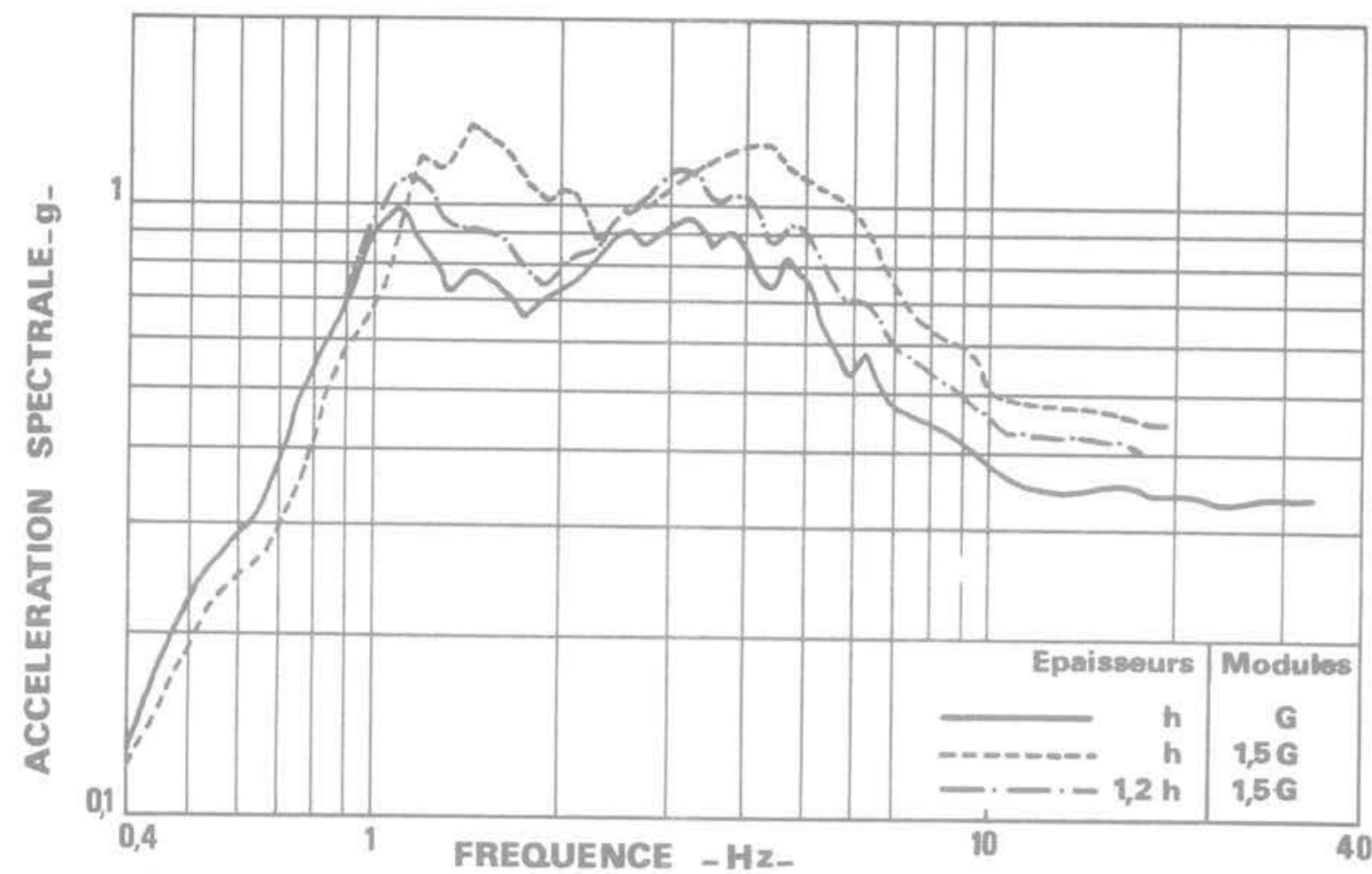

Fig. 19 Spectre de réponse à $5 \%$ d'amortissement critique à la surface du sol. Accélérogramme $0,30 \mathrm{~g}$ EDF

importante les hautes fréquences du mouvement. Le filtrage des hautes fréquences par la couche de sol existe toujours mais de façon nettement moins prononcée que ne le laisse supposer le calcul linéaire équivalent et à partir des fréquences plus élevées, de l'ordre de $7 \mathrm{~Hz}$. Ce filtrage des hautes fréquences est inhérent au calcul linéaire équivalent (Martin 1975) et provient du fait que les modules et amortissements du sol sont évalués sur la base d'une déformation moyenne contrôlée par les basses fréquences. II en résulte que les hautes fréquences, qui n'induisent que de faibles déformations, sont affectées d'un amortissement trop élevé.

Un des résultats nouveaux fourni par le calcul non linéaire est l'évaluation des déplacements de la surface du sol. Le déplacement relatif entre la surface et la base du profil de sol est donné sur la figure 18. Le déplacement maximal relatif au cours de la sollicitation est de $6 \mathrm{~cm}$ mais, plus intéressant, est l'apparition d'un déplacement résiduel de l'ordre du centimètre en fin de sollicitation. Ce déplacement résulte des non-linéarités du sol (déformation d'origine plastique) et ne peut bien entendu être évalué avec le modèle linéaire équivalent.

Les comparaisons effectuées montrent que dans les cas étudiés il est possible d'obtenir des résultats satisfaisants à l'aide du modèle linéaire équivalent. II est cependant probable que pour des niveaux d'accélération plus élevés les comparaisons ne seraient pas toujours aussi favorables comme d'autres études l'ont montré (Mohammadioun-Pecker, 1983).

\section{Influence des caractéristiques mécaniques du sol}

La figure 19 donne les spectres de réponse, à $5 \%$ d'amortissement critique du mouvement à la surface lorsque le module du sol est majoré de $50 \%$ en conservant l'épaisseur des couches constantes ou en majorant celles-ci de $20 \%$. La figure 20 présente les fonctions de transfert correspondant aux mêmes cas. Une augmentation de la raideur du sol à épaisseur constante conduit à des fréquences propres de la couche de sol plus élevées $(1,3 \mathrm{~Hz}$ au lieu de $1 \mathrm{~Hz}$ pour la fréquence fondamentale) et à une accélération maximale de surface plus élevée $(0,43 \mathrm{~g}$ au lieu de $0,32 \mathrm{~g}$ ). En milieu élastique, l'augmentation de raideur du sol devrait conduire à une augmentation de la fréquence fondamentale de $\sqrt{1,5} \simeq 1,2$.

L'augmentation calculée est plus importante car du fait de la non-linéarité du comportement, l'augmentation de raideur se traduit par des déformations induites plus faibles et donc par une réduction moins importante de la valeur du module. En se basant sur la fréquence fondamentale, l'augmentation de raideur effective du profil de sol est de $70 \%$ et non de $50 \%$.

De même, si on cherche à conserver la fréquence fondamentale de la couche de sol constante en se fondant sur les valeurs du module à petites déformations (majoration de $50 \%$ du module et de $20 \%$ de l'épaisseur), les spectres de réponse et fonction de transfert deviennent semblables à ceux du calcul initial. Une différence notable subsiste cependant due également au comportement non linéaire du sol.

Ces résultats montrent clairement qu'il n'existe pas, en toute rigueur, de notion simple comme la fréquence fondamentale d'une couche de sol. Du fait de la non-linéarité du sol, celle-ci dépend des déformations induites et donc en particulier du niveau de la sollicitation sismique. En conséquence, il apparaît illusoire de vouloir extrapoler des mesures de fréquences propres d'une couche de sol faites pour de faibles énergies mises en jeu (petits séismes, explosions de faible énergie...) à des secousses sismiques importantes. 


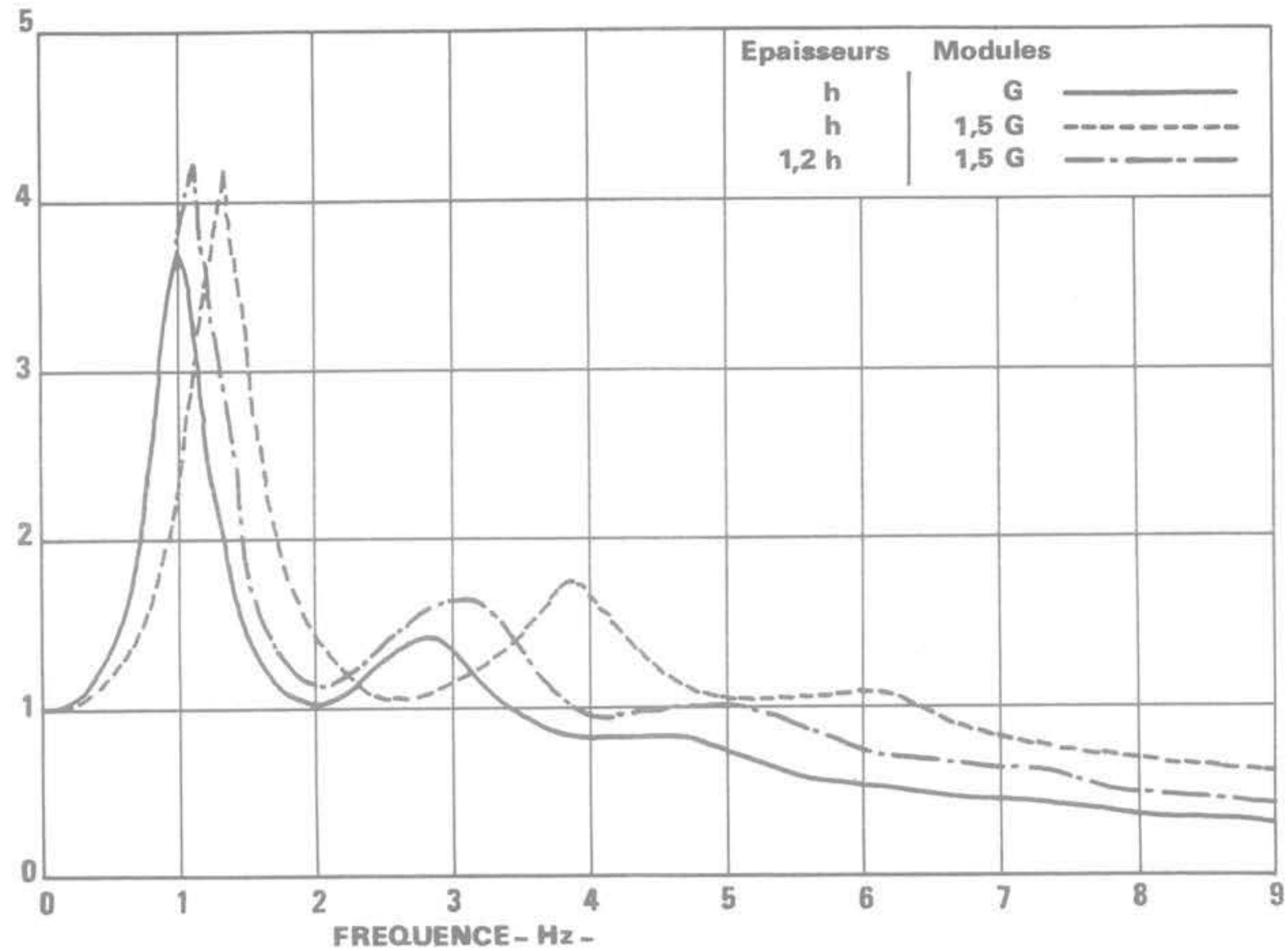

Fig. 20 Fonction de transfert du déplacement entre la base et la surface de la colonne de sol. Accélérations à l'affleurement $0,3 \mathrm{~g}$

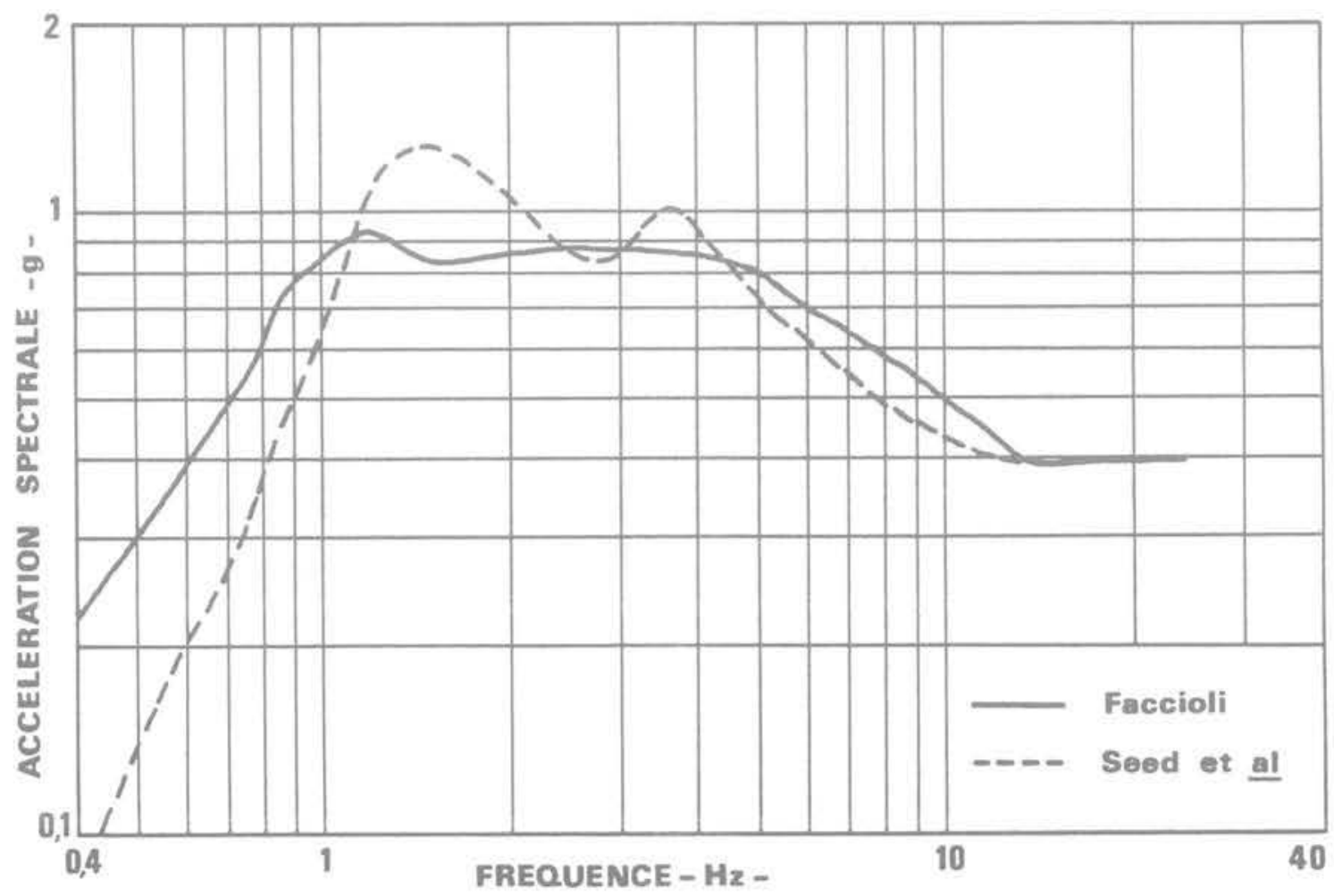

Fig. 21 Spectre de réponse à $5 \%$ d'amortissement critique sur sols mous 

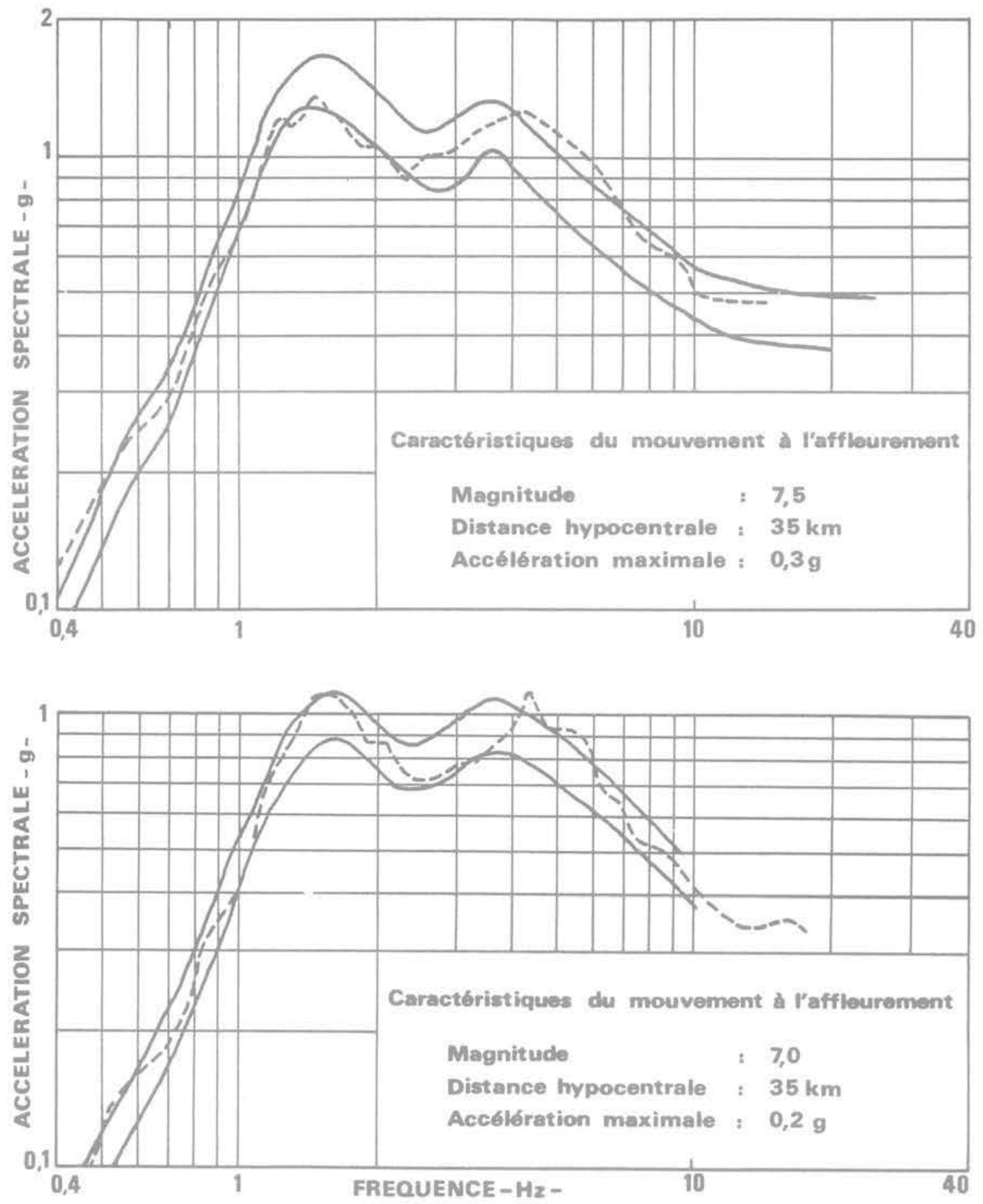

Fig. 22 Comparaison entre spectre à $5 \%$ d'amortissement critique calculé et enregistré à la surface du sol

\section{Comparaison avec des enregistrements réels}

Toutes les conclusions présentées précédemment résultent de calculs théoriques effectués de plus avec certaines hypothèses restrictives (nature des ondes par exemple). Il est donc intéressant et indispensable de comparer ces calculs aux quelques enregistrements réels disponibles. Ceux-ci, comme on l'a indiqué, sont peu nombreux dans le cas de sols mous. Les seules publications faisant état de tels enregistrements avec des renseignements suffisants sur la nature des sols sont à notre connaissance celles de Seed et al. (1976) et de Faccioli (1978).
Les premiers auteurs ont proposé un spectre de réponse normalisé pour sols mous. II n'est pas donné de relations entre le niveau de calage du spectre (accélération maximale) et divers paramètres caractéristiques du séisme (intensité, magnitude, distance focale...). Pour faciliter les comparaisons, on a retenu un niveau d'accélération maximale en surface égal à $0,39 \mathrm{~g}$. Le spectre moyen à $5 \%$ d'amortissement critique ainsi calé arbitrairement est donné sur la figure 21. II correspond à des sols constitués d'argile molle et de sable (15 enregistrements), La fréquence fondamentale moyenne des sites étudiés est de $0,8 \mathrm{~Hz}$ pour une accélération moyenne en surface de $0,055 \mathrm{~g}$ pour l'ensemble des enregistrements. Les épaisseurs 
de sol $(40 \mathrm{~m}$ à $200 \mathrm{~m})$ sont nettement plus importantes que celles considérées dans la présente étude.

Faccioli a donné à partir des 47 enregistrements, pour des sols caractérisés par une épaisseur d'une dizaine de mètres de matériau à vitesse de propagation des ondes de cisaillement inférieure à $100 \mathrm{~m} / \mathrm{s}$, un spectre de réponse défini par points : l'accélération spectrale est donnée à chaque fréquence en fonction de la magnitude et de la distance focale. On peut estimer d'après les lois d'atténuation de la plupart des auteurs qu'un séisme de magnitude 7,5 à 35 kilomètres de distance est susceptible de produire une accélération sur du rocher de l'ordre de $0,30 \mathrm{~g}$. On a donc retenu ces conditions sismiques pour tracer le spectre (fig. 21) proposé par Faccioli. L'accélération maximale de surface est dans ces conditions de $0,39 \mathrm{~g}$.

II ressort de l'examen de cette figure que :

- le spectre proposé par Seed et al., qui est un spectre lissé, ne fait pas apparaître nettement les singularités liées à la couche de sol en raison de la grande disparité des profils étudiés. Pour les fréquences supérieures à $3 \mathrm{~Hz}$, il est proche du spectre proposé par Faccioli, compte tenu du niveau de calage retenu. Pour les basses fréquences $(<1 \mathrm{~Hz})$ il est assez nettement enveloppe de ce dernier:

- le spectre proposé par Faccioli présente deux pics analogues à ceux obtenus dans nos calculs. Le second pic est cependant moins prononcé dans ce cas. L'examen des spectres de Faccioli calculés pour des conditions sismiques différentes de celles considérées montre qu'en général le second pic est aussi important que le premier (fig. 22). Les deux pics sont légèrement décalés vers des fréquences plus élevées que celles obtenues dans nos calculs pour le profil type. On a vu que la position de ces pics était liée aux fréquences propres de la couche de sol. Les profils examinés par Faccioli étant moins profonds (une dizaine de mètres) que celui de la présente étude, leurs fréquences propres sont plus élevées.

Les spectres obtenus par Faccioli (spectre moyen et spectre moyen plus un écart-type) sont comparés (fig. 22) à celui de la présente étude obtenu pour le cas du profil de sol le plus raide (modules majorés de $50 \%$, épaisseurs de couches constantes). Ce profil présente la particularité, par rapport au profil type, de conduire à des fréquences propres de la couche plus élevées, donc plus proches de celles des sites analysées par Faccioli. On constate que le spectre calculé se situe pratiquement pour toutes les fréquences entre les deux spectres de Faccioli qui, rappelonsle, proviennent d'enregistrements réels. La comparaison avec les spectres de Faccioli a également été effectuée pour d'autres conditions sismiques. Des lois d'atténuation relatives aux sites rocheux, on a déduit que l'accélération maximale de $0,2 \mathrm{~g}$ à l'affleurement était susceptible d'être produite par un séisme de magnitude 7 prenant origine à 35 kilomètres du site. Les spectres obtenus par le calcul et déduits des résultats de Faccioli sont donnés sur la figure 22. L'accord est de nouveau remarquable.

Ces résultats mettent en évidence le fait qu'il est possible à partir de calculs simples (unidimensionnels) de retrouver des résultats en très bon accord avec les données expérimentales. En particulier, les principales caractéristiques de la réponse dynamique d'un profil de sol mou sont bien mises en évidence.

\section{Conclusions}

Les calculs numériques réalisés dans le cadre de cette étude ont permis de mettre en évidence les principales caractéristiques de la réponse au séisme d'une couche de vase molle. En particulier, on a identifié l'existence, dans le spectre de réponse du mouvement calculé à la surface, de deux pics prononcés au voisinage des deux premières fréquences propres de la couche. II convient cependant de noter, qu'en raison du comportement non linéaire du sol, la fréquence propre n'est pas une caractéristique intrinséque de la couche de sol; elle est fonction de la sévérité de la sollicitation et décroît lorsque celle-ci augmente.

Parmi tous les paramètres qui conditionnent la réponse du mouvement en surface, la nature du mouvement sismique à l'affleurement et le choix de

Tableau 1

Caractéristiques moyennes des sols

\begin{tabular}{|c|c|c|c|c|c|c|}
\hline Caractéristiques & Nature & $\begin{array}{l}\text { Vase } \\
\text { argileuse }\end{array}$ & $\begin{array}{l}\text { Vase } \\
\text { silteuse }\end{array}$ & Sable & $\begin{array}{l}\text { alluvions } \\
\text { anciennes }\end{array}$ & Rocher \\
\hline Poids volumique apparent sec & $\mathrm{kN} / \mathrm{m}^{3}$ & 8 & 9 & 16 & 17 & - \\
\hline Teneur en eau & $\%$ & 80 & 70 & 25 & 24 & - \\
\hline Poids volumique total & $\mathrm{kN} / \mathrm{m}^{3}$ & 14,5 & 15,5 & 20 & 21 & 24 \\
\hline Indice de plasticité & $\%$ & 60 & 40 à 50 & 一 & 一 & - \\
\hline $\begin{array}{l}\text { Angle de cisaillement } \\
\text { intergranulaire }\end{array}$ & & 18 & 25 & 30 & 35 & - \\
\hline Cohésion intergranulaire & $\mathrm{kPa}$ & 0 & 0 & 0 & 0 & - \\
\hline Cohésion apparente & $\mathrm{kPa}$ & $1,3 h+5$ & $1,7 h+30$ & - & - & - \\
\hline $\begin{array}{l}\text { Module de cisaillement } \\
\text { dynamique maximal }\end{array}$ & (éq. 22) & & & & & \\
\hline $\mathrm{K}$ & & 200 & 340 & 1000 & 2000 & 54000 \\
\hline$\alpha$ & & 0,6 & 0,5 & 0,5 & 0,5 & 0 \\
\hline$k$ & & 0,4 & 0,3 & 0 & 0 & 0 \\
\hline $\begin{array}{l}\text { Pourcentage d'amortissement } \\
\text { critique maximal }\end{array}$ & $\%$ & 30 & 28 & 24 & 20 & 0 \\
\hline $\begin{array}{l}\text { Vitesse de propagation } \\
\text { des ondes de cisaillement }\end{array}$ & $\mathrm{m} / \mathrm{s}$ & - & - & - & - & 1500 \\
\hline
\end{tabular}


son accélération maximale sont les paramètres prépondérants. II est donc fondamental, si l'on suit la méthodologie de cette étude pour déterminer les caractéristiques du mouvement en surface du sol, de choisir avec le plus grand soin ces conditions sismiques : l'accélérogramme imposé à l'affleurement doit en particulier être représentatif d'un accélérogramme de rocher.

La méthodologie suivie constitue à notre avis une alternative à la pratique réglementaire permettant de définir le mouvement à la surface du sol. Cette dernière consiste à déterminer un niveau d'accélération maximale puis de caractériser le mouvement par un spectre de réponse calé à ce niveau. Ce spectre de réponse a soit une forme normalisée invariable (U.S.N.R.C.), soit une forme dépendante des conditions sismiques mais indépendantes de la nature du sol (C.E.A.-E.D.F.), soit parfois une forme dépendant de la nature du sol mais indépendante des conditions sismiques (Seed et al.). La présente étude montre que, même calés au niveau d'accélération approprié, ils ne rendent pas compte des particularités des sols mous; ils sous-estiment en particulier, dans la présente étude, les accélérations spectrales dans toute la plage des fréquences comprises entre $1 \mathrm{~Hz}$ et $4 \mathrm{~Hz}$. Ils les surestiment parfois fortement pour d'autres fréquences.

En conclusion, nous pensons que la méthodologie suivie permet de mieux rendre compte de la réalité et de traduire plus valablement les caractéristiques de la réponse au séisme des sols mous. Les comparaisons que nous avons pu effectuer avec des enregistrements réels le confirme de façon indiscutable et offrent des perspectives certaines pour I'utilisation de telles approches.

\section{Références bibliographiques}

[1] Devillers C. Mohammadioun (1981): «French methodology for determining site adapted SMS spectra *. SMIRT, 6th International Conference, Paris.

[2] Faccioli E. (1978) : « Response Spectra for soft soil sitesn. Earthquake Engineering and Soil Dynamics, ASCE Specialty Conference, Pasadena.

[3] Hayashi S., Tsuchida H., Kurata E. (1971): «Average response spectra for various subsoil Conditions». U.S. Japan panel on wind and seismic effects, Tokyo.
[4] Iwan W.D. (1967) : On a class of models for the yielding behavior of continuous and composite systems $*$. J. appl. mech. 34, 612-617.

[5] Jacobsen L. (1930): « Motion of a soil subjected to a simple harmonic ground vibration". Bull. seis. soc. Am. 20.

[6] Joyner W.B., Chen T.F. (1975): «Calculation of non-linear ground response in earthquakes $n$. Bull. seis. soc. Am. 65 1315-1336.

[7] Lysmer J. et al. (1975) : "Flush - A computer program for approximate $3 \mathrm{D}$ Analysis of soil-structure interaction problems $*$. EERC 75-30.

[8] Martin P.P. : "Non linear methods for dynamic analysis of ground response $*$. PhD Thesis, University of California, Berkeley, Cal.

[9] Masing G (1926) : « Eigenspannungen und Verfestigung beim Messing ". Proc. 2nd Int. Conf. of Applied Mechanics.

[10] Mohammadioun B., Pecker A. (1983): "On the limitation of the transmissibility of seismic energy by superficial soil deposits and soft rocks". Soumis à publication International Journal of Earthquake Engineering and structural dynamics.

[11] Mohraz B. (1976): «A study of earthquake response spectra for different geological conditions $\%$. Bulletin Seism-Soc. America 66, n०3 June.

[12] Dupas J.M., Pecker A. (1981) : "Étude expérimentale du comportement d'une vase sous chargement cycliquen. Revue française de Géotechnique 15, 59-73.

[13] Schnabel P., Seed H.B., Lysmer J. : " Shake - A computer program for earthquake response analysis of horizontally layered sites ". EERC Report 72-12, University of California, Berkeley, Cal.

[14] Seed H.B., Ugas C., Lysmer J. (1976): « Site dependent spectra for earthquarke resistant design $*$, Bulletin of Seismological Society of America, Vol. 66.

[15] Streeter V.L., Wylie E.B., Richard F.E. ; " Charsoil - Characteristics method applied to soils $n$. University of Michigan, Ann Arbor. 